Review

\title{
Nanosized and hierarchical zeolites: A short review
}

\author{
Esmat Koohsaryan, Mansoor Anbia* \\ Research Laboratory of Nanoporous Materials, Faculty of Chemistry, Iran University of Science and Technology, P.0. Box 16846-13114, Tehran, Iran
}

\section{A R T I C L E I N F O}

\section{Article history:}

Received 22 October 2015

Accepted 6 January 2016

Published 5 April 2016

\section{Keywords:}

Zeolite

Nanozeolite

Hierarchical zeolite

Catalyst

\begin{abstract}
A B S T R A C T
Zeolites are crystalline aluminosilicates with three-dimensional microporous structures. They have been used as ion-exchangers, catalysts, and adsorbents in various fields such as oil refining, petrochemistry, agriculture, and water and wastewater treatment. Their wide use is because of their many beneficial properties, such as framework and compositional flexibilities, physical and hydrothermal stabilities, non-toxicity, high surface areas, exchangeable cations, and good cost-benefit ratios. Although many zeolite applications depend on their microporous structures, this can cause diffusional constraints for bulky reactant and product molecules. There have been many efforts to overcome the intrinsic limitations of conventional zeolites by preparing nanosized and hierarchically structured zeolites. As a result of these efforts, several strategies have been established and the use of new zeolitic materials in various catalytic and adsorptive reactions has been investigated. Longer lifetimes, high catalytic performances, and postponed coking and catalyst deactivation can be achieved using hierarchical and nanosized zeolites. The aim of this review is to provide an overview of the enhanced properties of hierarchical and nanosized zeolites, and recent development methods for their synthesis. The advantages and disadvantages of each route are discussed, and the catalytic applications of nanozeolites and zeolites with secondary porosity, and a comparison with conventional zeolites, are briefly presented.
\end{abstract}

(C) 2016, Dalian Institute of Chemical Physics, Chinese Academy of Sciences. Published by Elsevier B.V. All rights reserved.

\section{Introduction}

Zeolites, glasses, carbons, and oxides are members of a large group of porous materials known as molecular sieves. Among these, zeolites are the most attractive candidates for industrial applications because of their unusual features. Many aspects of our daily lives are affected directly or indirectly by zeolites. Zeolites are crystalline materials with well-defined porous structures and pore sizes of molecular dimensions in the range 0.3-2 $\mathrm{nm}$; they are microporous solids, according to the IUPAC classification. They consist of $\mathrm{TO}_{4}$ tetrahedra $\mathrm{T}$ is $\mathrm{Al}$ or $\mathrm{Si}$ in a natural zeolite and can be other elements such as $\mathrm{Ti}, \mathrm{Ge}, \mathrm{P}$, and $B$ in synthetic ones), which are connected with each other by sharing corner oxygen atoms [1-6]. The final framework structure of a typical zeolite is obtained from secondary structures formed by primary tetrahedra [7].

Crystalline zeolites are usually prepared by hydro/solvothermal methods under appropriate conditions such as time and temperature of reaction, type of mineralizing agent, source of T atoms, structure-directing agents (SDAs), and calcination temperature [8-11]. In addition to three-dimensional channel systems occupied by water molecules and alkali or alkaline-earth metal cations, one- or two-dimensional zeolites are also possible [12]. Although synthetic zeolites are excellent choices for catalytic, adsorptive, and ion-exchange applications, inexpensive and abundant natural zeolites have not been widely used because of their impurities and structural defects [13]. Hydrophilic natural zeolites such as clinoptilolite, which

* Corresponding author. Tel: +98-2177240068; Fax: +98-2177241026; E-mail: anbia@iust.ac.ir 
have high cation-exchange capacities, have many benefits in water and wastewater treatment $[14,15]$. Natural zeolites also have applications in areas such as agriculture [16], gas separation [17], and animal husbandry [18]. Up to 2014, the Structure Commission of the International Zeolite Association (IZA-SC) had accepted 218 types of zeolite framework structure; other IZA structures may have been synthesized but not yet reported (Fig. 1) [19].

The most important applications of zeolites are in the oil-refining and petrochemical industries, in which synthetic zeolites with MFI, FAU, and $\beta$ frameworks have been commercialized as well-known catalysts [1]. The high selectivities of zeolites are important with respect to the requirements for certain petrochemical products. No corrosive and hazardous mineral acids are needed and fewer byproducts are formed [7].

The successful use of zeolites is based on their inherent shape-selective structures. There are three types of shape selectivity provided by the size and shape of zeolite inner vacancies. The first is reactant selectivity, which allows the introduction of certain small reactants into zeolite channels; the second is transition-state selectivity, in which intermediates of appropriate size are formed; and the third is product selectivity, enabling specific products to be obtained [20]. These features enable discrimination among molecules with regard to their size and shape, control of their passage through the porous system, and reactions with active sites. Although many excellent and unexpected results have been obtained using zeolites in a wide range of reactions, they cannot work properly in the presence of bulky molecules because such molecules cannot diffuse into the zeolite microporous structures; therefore, the efficiency and activity are lower in media containing such substrates. Only active sites close to the zeolite pore entrances or on their external surfaces, which represent at most $5 \%$ of the total number of active sites, are available for bulky molecules $[21,22]$. These constraints have stimulated research on zeolitic structures. Two main strategies have been developed to overcome these limitations, namely introducing secondary pores with diameters larger than $2 \mathrm{~nm}$ (meso- or macro-pores) and reducing the zeolite crystal size to the nanometer scale, to give

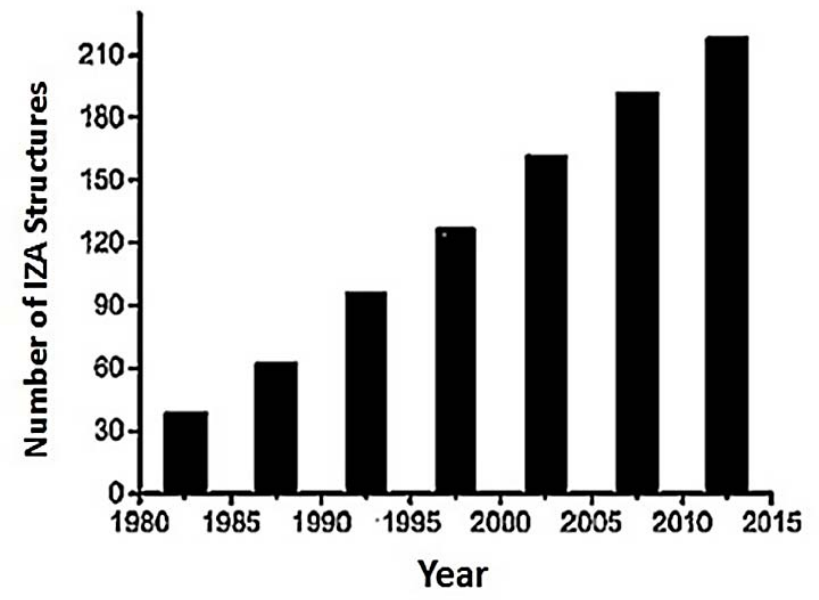

Fig. 1. Zeolite framework types recorded by IZA-SC. Reprinted with permission from Ref. [19]. Copyright 2014, American Chemical Society. hierarchical and nanosized zeolites, respectively. These improved structures with larger pores systems enhance diffusion of bulky reactant molecules into the pores and of large product molecules out of the pores. This shortens the distance to the active sites and facilitates adsorptive and catalytic reactions.

Fig. 2 shows different methods for nanozeolite and hierarchical zeolite synthesis from conventional zeolites. Some excellent reviews on hierarchical and nanosized zeolites have been published [23,24]. However, to the best of our knowledge, there is no review paper describing the properties and syntheses of both structures. The aim of this paper is to give a comprehensive review of zeolites and the improvements provided by hierarchical and nanosized structures; it will provide a good tutorial guide. We describe synthetic methods for both types of material (the syntheses of hierarchical structures are discussed in more detail than those of nanosized zeolites, in accordance with their importance) and briefly discuss their catalytic applications.

\section{Nanozeolites}

Since the beginning of the new millennium, much attention has been paid to nanosized porous materials [25]. Many chemical and physical processes involving a solid component are clearly affected by the differences between bulk and surface atoms in the solid. Increasing the amount of atoms exposed at solid surfaces with enhanced specific surface areas can be achieved by reducing the solid particle size or introducing an open pore system (Fig. 3) [26,27]. This is particularly important when zeolites are used as a main component of the reaction system in the form of adsorbents, ion-exchangers, or catalysts. As already mentioned, using zeolites with micron-diameter pores and micrometer-scale crystallites can hinder diffusion of bulky molecules because the extensive surface areas of the internal parts of the framework can only be accessed by molecules with kinetic diameters less than $1.5 \mathrm{~nm}$, and most natural and synthetic zeolites consist of micrometer-sized crystallites [28].

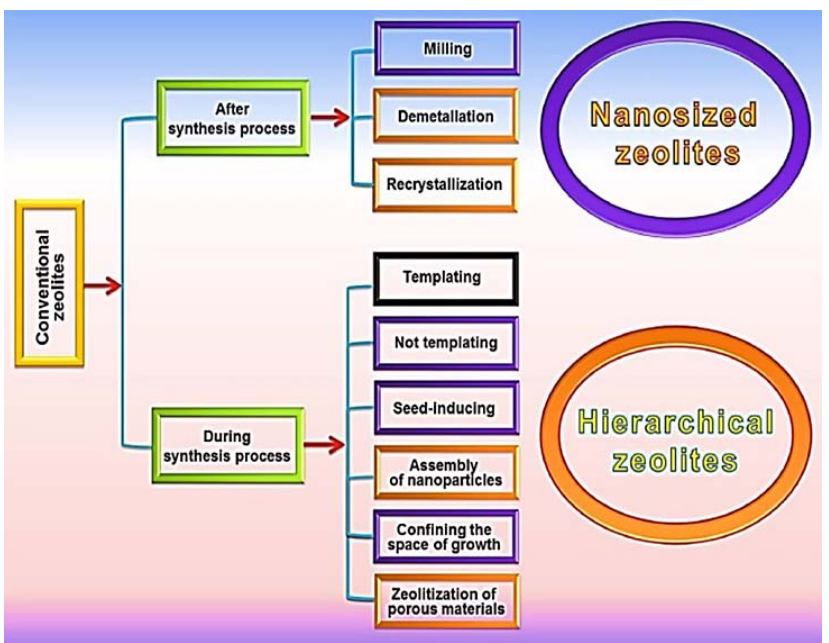

Fig. 2. Overview of synthetic methods for nanozeolites and hierarchical zeolites (colors indicate methods related to each type of zeolite; templating is used for both types). 


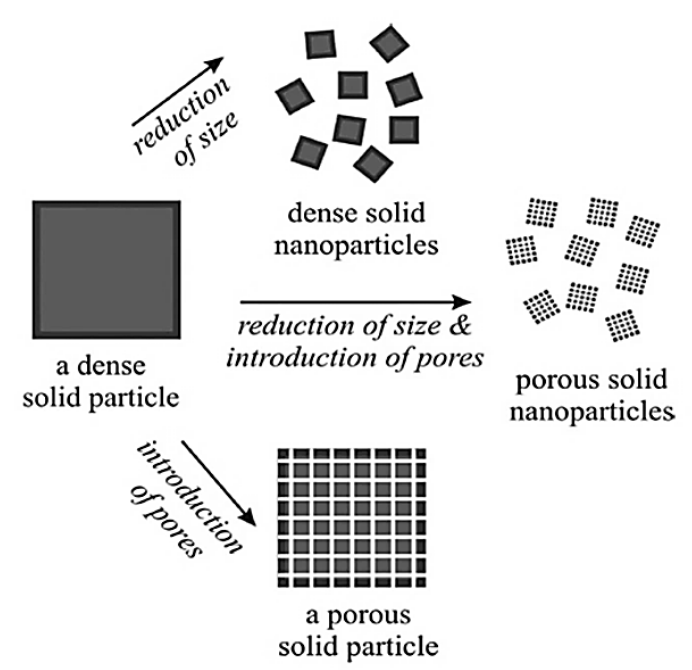

Fig. 3. Increasing surface areas of dense solids. Reprinted with permission from Ref. [26]. Copyright 2013, American Chemical Society.

The use of nanozeolites with crystal sizes less than $100 \mathrm{~nm}$ can overcome this limitation. Nanozeolites have properties such as enhanced surface areas, high external to internal atom number ratios, short diffusion path lengths, accessible active sites, and controllable surface properties, resulting in high activities, better performances, and longer lifetimes [29-31]. In the following sections, several approaches to nanocrystalline zeolite synthesis and catalytic applications of these materials are discussed.

\subsection{Methods for nanozeolite synthesis}

Nanozeolite synthesis has been widely studied in recent years. Ultradense gels, hydrogels, and clear solutions containing appropriate precursors can be used for zeolite synthesis. Control of the reaction conditions is important for producing nanosized zeolites. Nanozeolites with small crystal sizes are formed if the nucleation rate is faster than crystallite growth [26]. A zeolite framework can be obtained by arranging $\mathrm{AlO}_{4}{ }^{-}$ and $\mathrm{SiO}_{4}$ tetrahedra around positively charged species such as alkali metal cations or organic molecules as SDAs. The size, shape, and nature of the SDA affect formation of the zeolitic phase [32]. Tetraalkylammonium cations are the most popular SDAs and are usually used to prepare nanozeolites [33]. These templates modify the crystallite size and morphology and improve the crystallization efficiency. The type of tetraalkylammonium cation is responsible for producing a specific zeolite framework, e.g., tetramethylammonium hydroxide is an efficient template for LTA zeolite formation [34]. An attractive procedure for nanozeolite synthesis involves use of a combination of an inert porous matrix such as carbon black or carbon nanotubes and a conventional synthesis gel, resulting in growth into the enclosed spaces of voids with nanoparticle dimensions. The main factors that have an impact on the production of homogeneous particles are inertness, stability, and narrow pore size distribution of the matrix [24,26]. Investigation of the important synthetic parameters and optimization of the synthetic conditions (e.g., time, reaction temperature, and $\mathrm{Si}$ and Al sources) has helped to develop methods that increase the amount of nanosized nuclei formed, without using organic templates [32]. The use of harmful SDAs can be avoided by using a seed-assisted procedure in which a small amount of synthesized conventional zeolite seeds is added to an organic template-free synthesis gel, inducing formation of zeolitic structures with smaller particles [35-37]. These synthetic systems usually involve traditional hydro/solvothermal processes in which the heat and pressure required for the treatment are supplied using an autoclave and a heating oven. The use of unorthodox methods such as microwave and ultrasound irradiation to heat reaction vessels, the use of ionic liquids as green solvents and SDAs, and the use of microreactors have successfully produced nanozeolites with desirable properties [32,38-48]. Post-synthesis treatment involving milling of the primary zeolites with micrometer-sized particles, followed by recrystallization, also produces nanozeolites [49-52]. The physicochemical properties of some industrially important nanocrystalline zeolite catalysts are shown in Table 1 [26]. Here, the synthetic methods for nanozeolite preparation have been briefly stated; more information and a schematic diagram of each procedure are given in Table 2 and Fig. 4, respectively.

\subsection{Catalytic applications of nanozeolites}

Nanozeolites with enhanced performances are suitable successors to conventional micron-sized zeolites in many fields. Many studies have been devoted to evaluating the adsorptive and catalytic functions of nanozeolites in the removal of heavy metals [70], organic dyes [71], aromatics [72], and phenolic and phosphonate compounds [73,74] from aqueous solutions. They are also excellent catalysts in the petrochemical and oil-refining industries for successful conversion of feedstock to specific desired products in a wide range of reactions such as aromatization and catalytic cracking [75-79]. Nanozeolites with well-defined structures can combine significant Brönsted acidity and excellent mass transfer features; this improves the activity and efficiency of a catalytic reaction [80]. An example is nanocrystalline hydrothermally synthesized (emulsion method) ZSM-5, which has been used as a catalyst for cracking naphtha hydrocarbons ( $n$-hexane, cyclohexane, and methylcyclohexane) at atmospheric pressure and 823-923 $\mathrm{K}$ in a fixed-bed reactor [81]. In the case of $n$-hexane cracking, because macro- and nano-scale zeolites have similar acidities, the same initial yields were observed (about 83\%). However, over time, diffusion resistance in the macroscale catalyst, which induces more reactions, coking, and production of aromatics, was higher than in the case of nanozeolites, which have enhanced external surface areas and reduced diffusion resistance, resulting in stable activity and product selectivity. The conversion and product selectivity in the catalytic conversion of cyclohexane and methylcyclohexane (naphthene) were different from those in $n$-hexane cracking, because the primary products differed (Fig. 5). The performance of the macrosized catalyst decreased significantly with reaction time because dehydrogenation reactions such as methylcyclohexane to toluene and 
Table 1

Physicochemical properties of some industrially important nanosized zeolite catalysts. Reprinted with permission from Ref. [26]. Copyright 2013, American Chemical Society.

\begin{tabular}{|c|c|c|c|c|c|}
\hline $\begin{array}{l}\text { Microporous } \\
\text { material type }\end{array}$ & Molar composition of initial system & $\mathrm{T} /{ }^{\circ} \mathrm{C}$ & $\begin{array}{l}\text { Crystal size } \\
(\mathrm{nm})\end{array}$ & $\mathrm{Si} / \mathrm{Al}$ & Yield (\%) \\
\hline FAU & $9.6 \mathrm{Na}_{2} \mathrm{O}: 1.0 \mathrm{Al}_{2} \mathrm{O}_{3}: 14.4 \mathrm{SiO}_{2}: 198-334 \mathrm{H}_{2} \mathrm{O}$ & 60 & $34-56$ & 1.83 & 43 \\
\hline FAU & $5.0 \mathrm{Na}_{2} \mathrm{O}: 1.0 \mathrm{Al}_{2} \mathrm{O}_{3}: 5.0 \mathrm{SiO}_{2}: 10.0 \mathrm{H}_{2} \mathrm{O}$ & 90 & $120-200$ & na $^{\mathrm{e}}$ & na \\
\hline FAU & $0.032 \mathrm{Na}_{2} \mathrm{O}: 2.4(\mathrm{TMA})_{2} \mathrm{O}: 1.0 \mathrm{Al}_{2} \mathrm{O}_{3}: 3.4 \mathrm{SiO}_{2}: 370.0 \mathrm{H}_{2} \mathrm{O}$ & 100 & $20-30$ & 2.23 & na \\
\hline FAU & $0.048 \mathrm{Na}_{2} \mathrm{O}: 2.4 \mathrm{TMA}(\mathrm{OH}): 2.40 \mathrm{TMABr}: \mathrm{Al}_{2} \mathrm{O}_{3}: 4.35 \mathrm{SiO}_{2}: 249.0 \mathrm{H}_{2} \mathrm{O}$ & 100 & 96 & 2.25 & na \\
\hline FAU/EMT & $0.53 \mathrm{Li}_{2} \mathrm{O}: 3.0(\mathrm{TMA})_{2} \mathrm{O}: 0.5 \mathrm{Al}_{2} \mathrm{O}_{3}: 3.4 \mathrm{SiO}_{2}: 315.0 \mathrm{H}_{2} \mathrm{O}$ & 100 & $\sim 100$ & 3.17 & na \\
\hline EMT & $18.45 \mathrm{Na}_{2} \mathrm{O}: 1.0 \mathrm{Al}_{2} \mathrm{O}_{3}: 5.15 \mathrm{SiO}_{2}: 240.3 \mathrm{H}_{2} \mathrm{O}$ & $30^{\mathrm{a}}$ & $7-15$ & 1.14 & 65 \\
\hline EMT & $17.48 \mathrm{Na}_{2} \mathrm{O}: 1.0 \mathrm{Al}_{2} \mathrm{O}_{3}: 5.0 \mathrm{SiO}_{2}: 340.3 \mathrm{H}_{2} \mathrm{O}$ & & $50-70$ & & \\
\hline MFI & $9.12(\mathrm{TPA})_{2} \mathrm{O}: 0.5 \mathrm{Al}_{2} \mathrm{O}_{3}: 60.0 \mathrm{SiO}_{2}: 936.0 \mathrm{H}_{2} \mathrm{O}$ & 80 & $20-46$ & 60 & na \\
\hline MFI & 7.4 $\mathrm{Na}_{2} \mathrm{O}: 0.88 \mathrm{Al}_{2} \mathrm{O}_{3}: 25 \mathrm{SiO}_{2}: 1168 \mathrm{H}_{2} \mathrm{O}(0.1,1,3$ wt $\%$ seeds $)$ & $\begin{array}{l}100,120 \\
150,170\end{array}$ & $140-230$ & 10 & 92 \\
\hline MFI & $28.0 \mathrm{Na}_{2} \mathrm{O}: 1.0 \mathrm{Al}_{2} \mathrm{O}_{3}: 25 \mathrm{SiO}_{2}: 1168 \mathrm{H}_{2} \mathrm{O}$ (4 wt\% seeds) & 170 & $270-520$ & $16-22$ & na \\
\hline MFI & $x \mathrm{Na}_{2} \mathrm{O}: 1.0 \mathrm{Al}_{2} \mathrm{O}_{3}: 25 \mathrm{SiO}_{2}: 1168 \mathrm{H}_{2} \mathrm{O}(4 \mathrm{wt} \%$ seeds $)$ & 210 & $400-600$ & $10-18$ & 56 \\
\hline MFI & $0.5(\mathrm{TPA})_{2} \mathrm{O}: 3.0 \mathrm{SiO}_{2}: 52.4 \mathrm{H}_{2} \mathrm{O}: 25.1 \mathrm{EtOH}+$ methylene blue & 100 & $40-60$ & $\infty$ & na \\
\hline MFI & $0.25(\mathrm{TPA})_{2} \mathrm{O}: 1.0 \mathrm{SiO}_{2}: 0.016 \mathrm{~L}$-glu: $11.0 \mathrm{H}_{2} \mathrm{O}$ & 170 & 60 & $69 \mathrm{~d}$ & na \\
\hline MFI & $0.23(\mathrm{TPA})_{2} \mathrm{O}: 0.033 \mathrm{TiO}_{2}: 1.0 \mathrm{SiO}_{2}: 0.016 \mathrm{~L}$-glu: $11.0 \mathrm{H}_{2} \mathrm{O}$ & 170 & 100 & 81,95 & \\
\hline BEA & $0.35 \mathrm{Na}_{2} \mathrm{O}: 4.5(\mathrm{TEA})_{2} \mathrm{O}: x^{\mathrm{b}} \mathrm{Al}_{2} \mathrm{O}_{3}: 25 \mathrm{SiO}_{2}: 295 \mathrm{H}_{2} \mathrm{O}$ & 100 & $30-50$ & $14,23,32,42, \infty$ & na \\
\hline BEA & $0.15 \mathrm{TMP}(\mathrm{OH})_{2} \mathrm{c}^{\mathrm{a}} 1.0 \mathrm{SiO}_{2}: 25 \mathrm{H}_{2} \mathrm{O}$ & 100 & 140,200 & $\infty, 35$ & na \\
\hline BEA & $7.5 \mathrm{Na}_{2} \mathrm{O}: 0.75 \mathrm{Al}_{2} \mathrm{O}_{3}: 25 \mathrm{SiO}_{2}: 375 \mathrm{H}_{2} \mathrm{O}(2.5 \mathrm{wt} \%$ seeds $)$ & 100 & 400 & $3.9-26$ & $10-40$ \\
\hline BEA & $0.18(\mathrm{TEA})_{2} \mathrm{O}: 0.02 \mathrm{Al}_{2} \mathrm{O}_{3}: 1.0 \mathrm{SiO}_{2}: 5.6 \mathrm{H}_{2} \mathrm{O}$ & 90 & 57 & $18-21$ & 60 \\
\hline MOR & $\mathrm{Na}_{2} \mathrm{O}: \mathrm{Al}_{2} \mathrm{O}_{3}: 10 \mathrm{SiO}_{2}: 48 \mathrm{H}_{2} \mathrm{O}$ & 175 & $10-50$ & 5 & na \\
\hline MOR & $0.275 \mathrm{Na}_{2} \mathrm{O}: 0.025-0.06 \mathrm{Al}_{2} \mathrm{O}_{3}: 1.0 \mathrm{SiO}_{2}: x \mathrm{H}_{2} \mathrm{O}$ & $140-170$ & $500-700$ & $11-21$ & na \\
\hline
\end{tabular}

${ }^{a}$ Conventional and microwave $(200 \mathrm{~W})$ heating at $30^{\circ} \mathrm{C} .{ }^{\mathrm{b}} \mathrm{X}=0,0.05,0.15,0.25$, and $0.50 .{ }^{\mathrm{c}} \mathrm{TMP}(\mathrm{OH})_{2}=4,4^{\prime}$-trimethylenebis $(N$-methyl- $N$-benzylpiperidinium)dihydroxide. ${ }^{\mathrm{d}} \mathrm{Si} / \mathrm{Ti}$ ratio. ${ }^{\mathrm{e}}$ na $=$ not available.

Table 2

Methods for nanosized zeolite synthesis.

\begin{tabular}{|c|c|c|c|}
\hline Synthesis Methods & Characteristics & Advantages/Difficulties & [Ref] \\
\hline Templating & $\begin{array}{l}\text { Using organo-templates to direct the certain } \\
\text { zeolite framework structure }\end{array}$ & $\begin{array}{l}\text { Simplicity and getting desired zeolitic phase with ho- } \\
\text { mogenous nanoparticles/environmental and economic } \\
\text { issues in accordance to SDA and time consumption }\end{array}$ & {$[32-34,53-57]$} \\
\hline Not-templating & $\begin{array}{l}\text { Optimization of the reaction conditions (time, } \\
\text { temperature, type of precursors) while not using } \\
\text { templates }\end{array}$ & Capability for scaling-up/ thorough tuning the conditions & {$[58-62]$} \\
\hline Milling & Mechanical grinding of initial zeolite materials & Simplicity and eco-friendly/loss of some crystalline parts & [49-52] \\
\hline Seed-inducing & $\begin{array}{l}\text { Adding small amount of zeolitic seeds to the } \\
\text { free-template synthetic gel }\end{array}$ & $\begin{array}{l}\text { Desired zeolitic phase with high yield/ truly seed intro- } \\
\text { duction }\end{array}$ & {$[35-37,63,64]$} \\
\hline $\begin{array}{l}\text { Confining the } \\
\text { space of growth }\end{array}$ & $\begin{array}{l}\text { Using an inert matrix with constrained regions } \\
\text { to allow nanoparticles to grow therein }\end{array}$ & $\begin{array}{l}\text { Getting product with certain particle size/high combus- } \\
\text { tion temperature and probability of structural damage }\end{array}$ & [65-68] \\
\hline Alternatives routes & $\begin{array}{l}\text { Altering the conventional solvents, heating } \\
\text { system and reaction chamber using microwave } \\
\text { and ultrasounds irradiation, green solvents like } \\
\text { ionic liquids and low-volume microreactors }\end{array}$ & $\begin{array}{l}\text { Enhanced interaction between reactants during shorter } \\
\text { time and lower temperature resulting in uniform nano- } \\
\text { particles and eco-friendly processes due to absence or } \\
\text { low extent of SDAs or solvents/economic and } \\
\text { self-protection issues }\end{array}$ & {$[39-46,48,69]$} \\
\hline
\end{tabular}

cyclohexane to benzene easily occurred in addition to the cracking reaction; these all stem from resistance to molecular diffusion. Deactivation of macrosized zeolites was therefore higher in naphthene cracking than in $n$-hexane conversion.

However, outstanding stability for propylene (a stable propylene/ethylene carbon molar ratio of approximately 2.0) and high light olefin yields were achieved using a nanoscale ZSM-5 zeolite. The performance of the nanoscale catalyst was not affected by the reactant type. The viability of the ZSM-5 zeolite and the effects of its crystal size on the dehydration reaction of methanol and product distribution were also assessed in a fixed-bed continuous flow reactor at a weight hourly space 


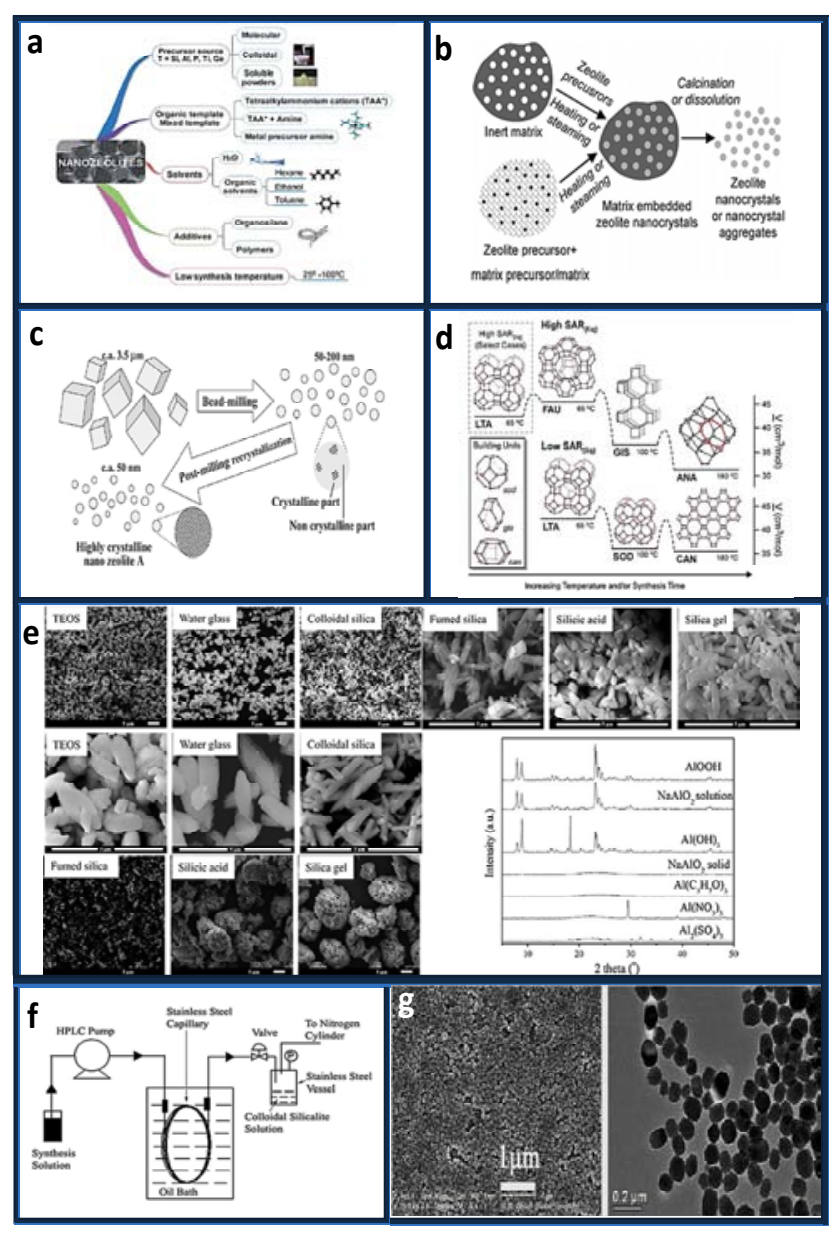

Fig. 4. Schematic diagrams of various methods for nanozeolite synthesis. (a) Main factors affecting zeolite nanocrystal formation. Reprinted with permission from Ref. [32]. Copyright 2013, Royal Society of Chemistry. (b) Schematic diagram of confined-space method for synthesizing zeolite nanocrystals. Reprinted with permission from Ref. [24]. Copyright 2005, American Chemical Society. (c) Bead-milling and post-milling recrystallization method for nanozeolite synthesis. Reprinted with permission from Ref. [50]. Copyright 2011, American Chemical Society. (d) Phase transformation of Na zeolite with increasing synthesis temperature and/or time. Reprinted with permission from Ref. [59]. Copyright 2013, American Chemical Society. (e) SEM images and XRD patterns of samples synthesized by organic-template-free method using various $\mathrm{Si}$ and $\mathrm{Al}$ sources. Reprinted with permission from Ref. [60]. Copyright 2012, Elsevier. (f) Synthesis of silicalite nanocrystals using a pressurized capillary microreactor. Reprinted with permission from Ref. [69]. Copyright 2009, John Wiley and Sons. (g) SEM and TEM images of ZSM-5 nanocrystals synthesized at $110^{\circ} \mathrm{C}$ using microwave-assisted method. Reprinted with permission from Ref. [41]. Copyright 2009, Elsevier.

velocity of $2.6 \mathrm{~g} /(\mathrm{g} \cdot \mathrm{h}), 370{ }^{\circ} \mathrm{C}$, and ambient pressure [82]. The conversion achieved with the conventional zeolite was $50 \%$, whereas $100 \%$ conversion was achieved using the nanosized catalyst (Fig. 6). The high total pore volume of the nanozeolite, which is related to the presence of multimodal porosity in its minute crystals, significantly increased the reaction activity and paraffin (C1-C4) and light olefin (propylene and ethylene) selectivities; the total pore volume of the conventional zeolite is much smaller. The increase in the number of strong acid sites (a)

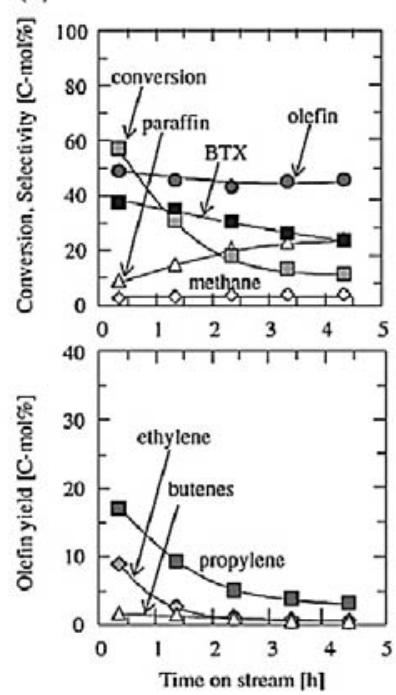

(b)
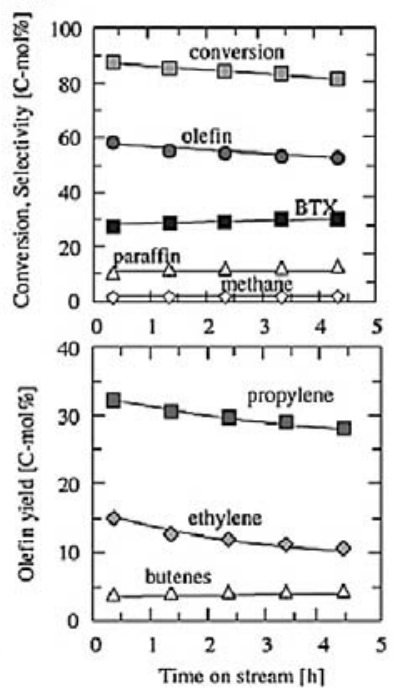

Fig. 5. Product selectivities, conversions, and light olefin yields in methylcyclohexane cracking over (a) macroscale and (b) nanoscale ZSM-5. Reprinted with permission from Ref. [81]. Copyright 2013, Elsevier.

available for the reactants on the surfaces of uniform nanocrystals prevent diffusion constraint and accelerate the catalytic conversion reaction. In contrast, catalysts with lower Brunauer-Emmett-Teller (BET) surface areas (and therefore fewer strong acid sites) severely inhibit molecule access to the active sites of conventional zeolites, and extensive methanol dehydration cannot be achieved.

In addition to catalytic applications, nanozeolites have attracted much attention for use in zeolite films and membranes, fuel cells, microreactors, micro/nanoelectronics, (bio)sensors, and polymer-zeolite nanocomposites [83].

As mentioned above, many strategies for nanozeolite synthesis have been explored. Generally, nanozeolites are in the form of dispersed colloidal suspensions and tend to agglomerate spontaneously. A high pressure drop when using a catalyst powder and difficulties in separating the catalyst from the reaction products are serious issues in nanozeolite handling. The conventional processes for solving these problems adversely affect the most important properties of nanomaterials, i.e., the active external surface. Hierarchical assembly appears to be the best method for meeting operating requirements and retaining the intrinsic properties of nanozeolites, and provides novel multiporous systems and functionality in one structure [84].

\section{Hierarchical zeolites}

Hierarchical porous materials, which have porous structures with multiple porosity levels (micro-, meso-, and macro-pores), have been the center of interest for the last decade. Micro- and meso-pores of uniform size are responsible for the size and shape selectivities for guest molecules and improving interactions between host and guest molecules, whereas macropores promote molecular diffusion and accessibility to 

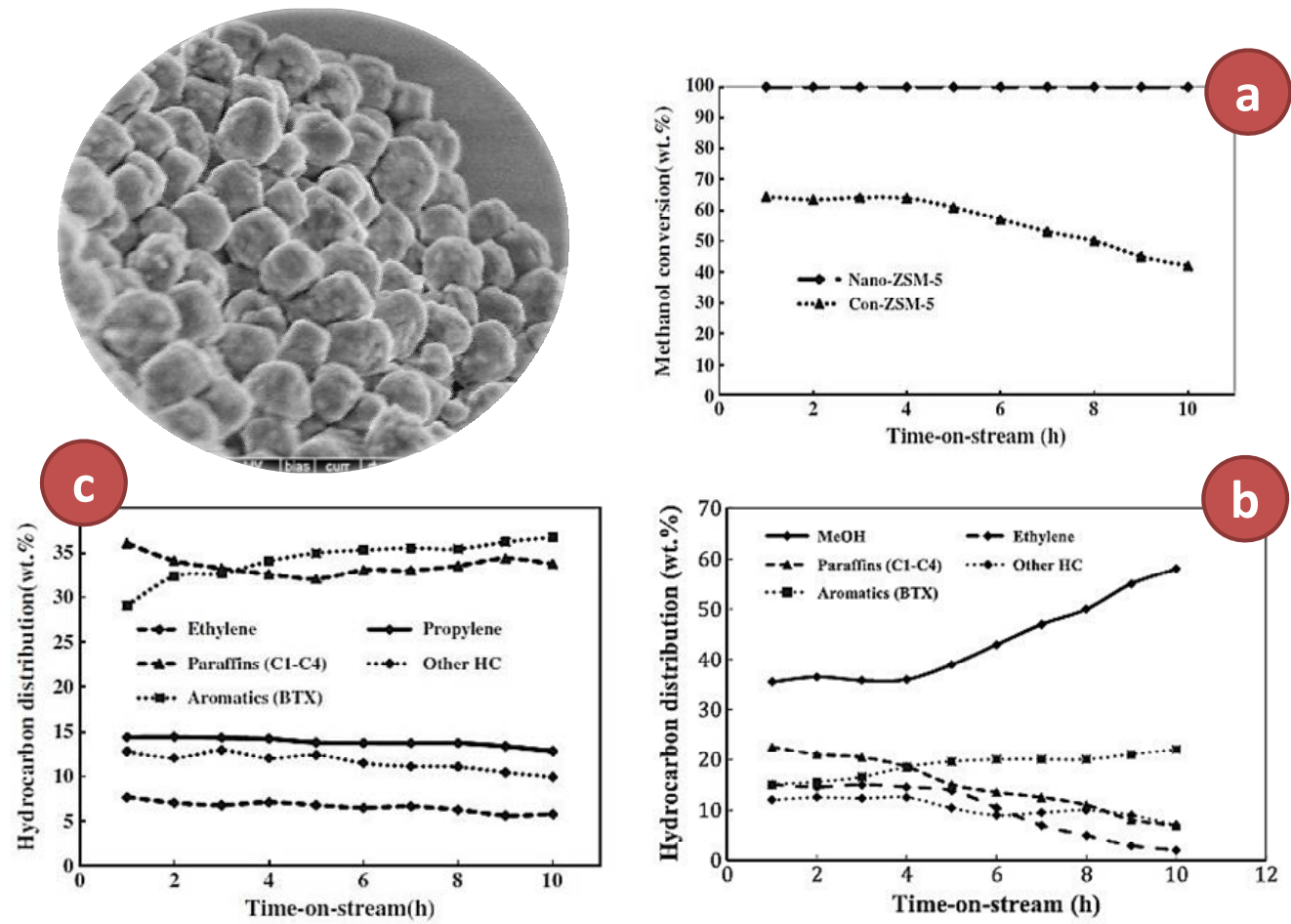

Fig. 6. XHR-SEM image of uniform ZSM-5 single nanocrystals and methanol conversion (a), and hydrocarbon distributions obtained from methanol dehydration over conventional ZSM-5 (b) and nano-ZSM-5 (c). Reprinted with permission from Ref. [82]. Copyright 2011, Elsevier.

active sites [85]. Hierarchical zeolites have additional porosity, i.e., meso- and/or macro-pores, in addition to their intrinsic micropores. Bimodal macro- microporous zeolites have been synthesized. Zeolites containing pores of diameter $2-50 \mathrm{~nm}$ in addition to their innate microporous systems are called hierarchical or mesoporous zeolites [86]. In a conventional zeolitic structure, because of configurational diffusion, the intracrystalline transportation of pervading species is the rate-controlling step in many catalytic and adsorption processes. Hierarchical zeolites can eliminate diffusion restrictions for large reactants and products. It could be concluded that because of the reduced lengths of diffusion pathways, hierarchical structures give no compound selectivity, but it has been reported that the chemical nature of the active sites controls the selectivity and product distribution in many reactions. Additionally, because product molecules can move away from the pores in these structures, coke precursors do not block the pore mouths, therefore catalyst deactivation is postponed and there are fewer side reactions and byproducts. However, it must be noted that although hierarchical zeolites are much more resistant than porous ones to deactivation by coking, the growth of external coke deposits can also block the secondary pores. Mesoporosity also enhances the zeolite surface area. Hierarchical structures also promote homogeneous dispersion of active components such as metals or oxides on the catalyst surfaces. Because of these improved properties and their intrinsic hydrothermal stabilities and strong acidities, mesoporous zeolites are important commercial catalysts [21,87]. Researchers have explored many routes for the synthesis of hierarchically structured zeolites. In general, these strategies can be classified into two groups. In the first approach, one zeolitic structure is prepared, and then post-synthesis treatments are used to introduce larger pores into the framework structure. This is a destructive or top-down method involving demetallation (desilication and dealumination) and recrystallization with the aid of surfactants. The other route involves introducing mesoporosity into the zeolitic structure from the beginning of the zeolite production process. The second method is a constructive or bottom-up method and includes mesostructured zeolite preparation with hard and soft templates, and assembling nanozeolites and converting the initial mesoporous materials into zeolites. Each category consists of several individual methods, which have both advantages and disadvantages. In the following part of this review article, we describe the synthetic procedures and catalytic applications of hierarchical zeolites.

\subsection{Synthesis of hierarchical zeolites}

\subsubsection{Synthesis of hierarchical zeolites by demetallation}

In the demetallation method, one constituent is preferentially extracted from a preformed zeolite to create secondary porosity in the structure, e.g., desilication and dealumination of the zeolite framework [88]. Dealumination is an effective method for synthesizing zeolites with large pores and has been used in industry for many years. Mineral and organic acid solutions, various chemicals, and high temperature during calcination and steaming can be used to eliminate $\mathrm{Al}$ species from the framework. In all cases, $\mathrm{Si}-\mathrm{O}-\mathrm{Al}$ bonds are hydrolyzed to decrease the Al content [23]. The first step after preparing the desired zeolite is calcination, in which some $\mathrm{Al}$ atoms are released from the framework, depending on the temperature and type of zeolite. Steam treatment at high temperature is a 
well-established method for adjusting the acidity of a zeolite [89]. Otomo et al. [90] assessed the influence of calcination and steam treatment on $\beta$ zeolite dealumination. In the first stage, the ammonium form of the $\beta$ zeolite was calcined and steam-treated at various temperatures between 500 and 750 ${ }^{\circ} \mathrm{C}$ and $500-600{ }^{\circ} \mathrm{C}$, respectively. The results showed that increasing the calcination temperature led to a reduction in the number of tetrahedral $\mathrm{Al}$ species related to Brönsted acid sites, and increased the number of Lewis acid sites. Fig. 7 shows that the intensities of the two peaks, at 0 and $57 \mathrm{ppm}$, related to extra-framework $\mathrm{Al}$ and tetrahedral $\mathrm{Al}$ atoms, respectively, in the magic-angle spinning nuclear magnetic resonance (MAS NMR) spectra decreased with increasing calcination and steam-treatment temperatures.

Weak Lewis acid sites were generated at high calcination temperatures and the total amount of acid sites decreased slightly. The treatment temperature had a greater effect on the acidic properties of the steam-treated samples than on the calcined samples, because dealumination of the steam-treated samples was more severe at a given temperature than that of the calcined samples.

Leaching the solid with mild acids helps to remove extra-framework $\mathrm{Al}$ atoms. Mineral acids such as hydrochloric, sulfuric, and nitric acids, and organic acids such as tartaric and oxalic acid, at appropriate concentrations, act as hydrolyzing agents [91]. The types of acid and zeolite are important. Dealumination of $\beta$, mordenite (MOR), and ZSM-5 zeolites with 6-mol/L HCl solution showed that the efficiency of the process was a function of the zeolitic structure; the efficiency order is $\beta$
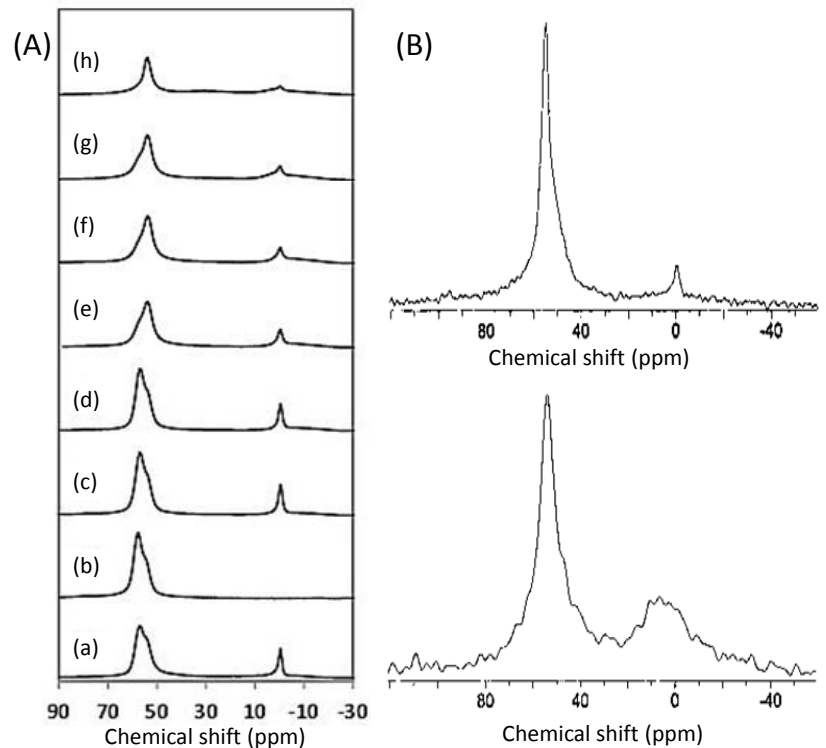

Fig. 7. (A) ${ }^{27} \mathrm{Al}$ MAS NMR spectra of dealuminated $\beta$ zeolites obtained by calcination and steam treatment at various temperatures. (a) Original $\beta$ zeolite calcined at $580{ }^{\circ} \mathrm{C}$ to remove SDA ( $\beta$-SDAcal); (b) Ammonium form of $\beta$-SDAcal $\left(\beta-\mathrm{NH}_{4}\right)$; (c) $\beta$ - $\mathrm{NH}_{4}$ calcined at $500{ }^{\circ} \mathrm{C}$; (d) $\beta-\mathrm{NH}_{4}$ calcined at $600{ }^{\circ} \mathrm{C}$; (e) $\beta-\mathrm{NH}_{4}$ calcined at $700{ }^{\circ} \mathrm{C}$; (f) $\beta-\mathrm{NH}_{4}$ calcined at $750{ }^{\circ} \mathrm{C}$; (g) $\beta-\mathrm{NH}_{4}$ steam-treated at $500{ }^{\circ} \mathrm{C}$; (h) $\beta-\mathrm{NH}_{4}$ steam-treated at $600{ }^{\circ} \mathrm{C}$. Reprinted with permission from Ref. [90]. Copyright 2014, Elsevier. (B) ${ }^{27} \mathrm{Al}$ MAS NMR spectra of mordenite zeolites treated with 6-mol/L nitric acid (top) and 2-mol/L oxalic acid (bottom). Reprinted with permission from Ref. [93]. Copyright 2000, Elsevier.
> MOR > ZSM-5 (Fig. 8) [92]. The order can be explained based on the flexibilities of the zeolite frameworks and the pore sizes and arrangements, which determine the accessibility to $\mathrm{Al}$ atoms. MOR and ZSM-5 are less flexible than $\beta$ zeolite, so they are more difficult to dealuminate. In addition, during acid leaching of MOR samples using 1- and 2-mol/L solutions of oxalic acid, the $\mathrm{Si} / \mathrm{Al}$ ratio increased to 120 , whereas the $\mathrm{Si} / \mathrm{Al}$ ratio obtained using 2- or 6-mol/L nitric acid solutions was about 70 [93]. These differences are attributed to oxalic acid acting as both a hydrolyzing and chelating agent, forming trioxalato $\mathrm{Al}$ complexes, with a high complexation constant. The acidity of the solution causes Al-O-Si bond hydrolysis, and then the Al cations are chelated by three oxalic acid molecules (Fig. 7). The formation of secondary (meso and partly macro) porous systems was observed with nitric acid. The high acidity of the nitric acid solution not only causes lattice defects, which weaken the structure locally during dealumination, but also dissolves the silica units; this does not occur in the case of oxalic acid at a lower $\mathrm{pH}$.

Chemicals that can substitute $\mathrm{Si}$ atoms for $\mathrm{Al}$, such as $\mathrm{SiCl}_{4}$ and ammonium hexafluorosilicate, induce mesopores in addition to the micropores of the parent zeolite. They have the benefit of directly replacing $\mathrm{Si}$ with released $\mathrm{Al}$ atoms and do not result in the formation of new hydroxyl nests and extra-framework $\mathrm{Al}$ species such as $\mathrm{Al}(\mathrm{OH})_{3}, \mathrm{Al}(\mathrm{OH})_{2^{+}}, \mathrm{Al}(\mathrm{OH})^{2+}$, and $\mathrm{Al}^{3+}$, which can clog pores and channels; instead, they heal pre-existing defects $[94,95]$. Chelating agents such as ethylenediaminetetraacetic acid (EDTA) that form complexes with Al cations are other options for zeolite dealumination. The rate of $\mathrm{H}_{4}$ EDTA addition to the zeolite suspension in water determines the nature of the dealuminated product, because removal of large amounts of $\mathrm{Al}$ without filling the vacancies caused by rapidly increasing the amount of $\mathrm{H}_{4}$ EDTA can cause collapse of the zeolite framework [96]. In this strategy, the Al present on the external surface is removed preferentially, giving rise to non-uniform $\mathrm{Al}$ distributions within the crystals [21]. Finally, it should be remembered that dealumination will change the $\mathrm{Si} / \mathrm{Al}$ ratio and acidity of the zeolite, with concurrent mesopore formation within the framework structure. Interpretation of the catalytic data is consequently difficult, because it is not clear whether the catalytic performance of the zeolite results from alterations in its acidity or the introduction of a new mesoporous system into the framework [91].

Demetallation is not limited to dealumination. Desilication is easy and uses inexpensive materials such as basic solutions

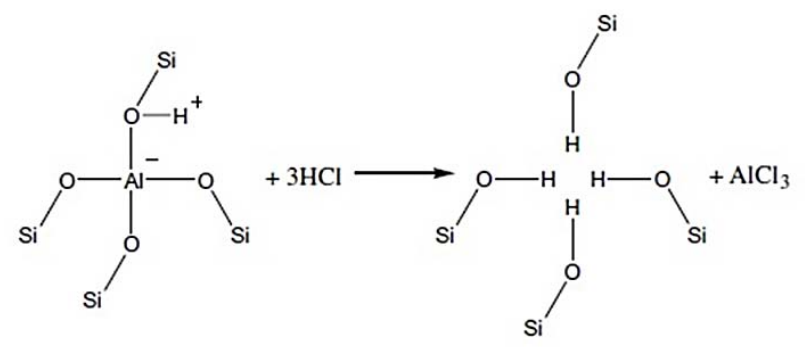

Fig. 8. Generation of silanol groups during dealumination in acidic medium. Reprinted with permission from Ref. [92]. Copyright 2011, Elsevier. 
(usually $\mathrm{NaOH}$ solution) to extract silica from the zeolite framework. Post-synthesis modification through base-leaching treatment to improve the adsorptive and catalytic performances of zeolites was first patented by Young in the 1960s [97]. Bonilla et al. [98] studied the desilication of a commercial ferrierite sample $(\mathrm{Si} / \mathrm{Al}=29)$ using $\mathrm{NaOH}$. Alkaline treatment of the zeolite was performed in a 16-parallel-reactor system using various $\mathrm{NaOH}$ concentrations (0.1-1 mol/L), times $(0.5-9 \mathrm{~h})$, and temperatures (333-363 K). All the T-sites in ferrierite are bonded to five rings, and therefore the framework $\mathrm{Al}$ species are highly stable and are less easily desilicated in an alkaline environment. The basic conditions used to treat other frameworks such as MFI, MTW, and MOR with similar Si/Al ratios would not be suitable for $\mathrm{Si}$ extraction from the ferrierite framework. These results show that both micro- and meso-porosities (inter- and intra-crystalline) were present in the alkali-treated samples, and they had a broad distribution of large mesopores, not a specific mesopore size, because of the peculiar morphology of the ferrierite crystals, which consist of thin platelets (50-150 nm). The mesopore surface areas of the samples desilicated under the optimum conditions in aqueous $\mathrm{NaOH}$ solution were three to four times higher than those of the dealuminated parent zeolites obtained by steaming and acid leaching; the intrinsic acidity and crystallinity were largely protected (Fig. 9).

Unlike ferrierite, in the case of $\beta$ zeolite, Al cannot moderate Si extraction, because of the relatively low stability of $\mathrm{Al}$ in the $\beta$ zeolite framework, therefore desilication will not effectively enhance the catalytic properties of zeolites with the BEA framework; methods such as hard-templating are better for creating mesoporosity [99]. To achieve a uniform distribution of mesopores, it is essential to use a certain range of $\mathrm{Si} / \mathrm{Al}$ ratios for the zeolite. The influence of the $\mathrm{Si} / \mathrm{Al}$ ratio of the zeolite on the alkaline treatment efficiency was evaluated for the first time by Groen and coworkers [100], in the desilication of commercial ZSM-5 zeolites covering a broad range of nominal molar Si/Al ratios from 15 to 1000. The Si/Al ratio range from 25 to 50 was identified as a prerequisite for rich desilication; framework $\mathrm{Al}$ species act as a pore-directing agent (PDA) because they control Si removal [97]. Abelló et al. [101] suggested that the use of strong organic bases such as tetrapropylammonium or tetrabutylammonium hydroxides (TPAOH or TBAOH) as desilicating agents avoids the additional ion-exchange step needed with inorganic bases to obtain $\mathrm{H}$-form zeolites. Hierarchical zeolites obtained in the presence of strong inorganic bases such as $\mathrm{NaOH}, \mathrm{KOH}$, and $\mathrm{LiOH}$ can undergo exchange of their $\mathrm{NH}_{4}{ }^{+}$or $\mathrm{H}^{+}$with extra $\mathrm{Na}^{+}, \mathrm{K}^{+}$, or $\mathrm{Li}^{+}$cations in the alkaline solution. An additional ion-exchange process with $\mathrm{NH}_{4} \mathrm{NO}_{3}$ solution followed by calcination is therefore needed to obtain the frequently favorable protonic form of the mesoporous zeolite. The high yields achieved with treatments using organic hydroxides (ca. 95\% for TPAOH compared with $62 \%$ for $\mathrm{NaOH}$ ) imply that the reactions can be controlled because of the relatively low solubilities of silicones in organic hydroxides, whereas the fast creation of a hierarchical system within the zeolitic structure using $\mathrm{NaOH}$ solution does not allow precise adjustment of the conditions (Fig. 10).

In summary, the main advantage of desilication is its simplicity and inexpensive starting materials. However, partial damage of the zeolitic structure and loss of micropores and crystallinity can occur, therefore the treatment should be precisely controlled to prevent formation of amorphous parts or extra-framework Al [21]. Pretreatment of zeolites or using low-temperature calcined zeolites with less-damaged structures and use of a surfactant to help recrystallization are rational strategies for overcoming these problems [91].
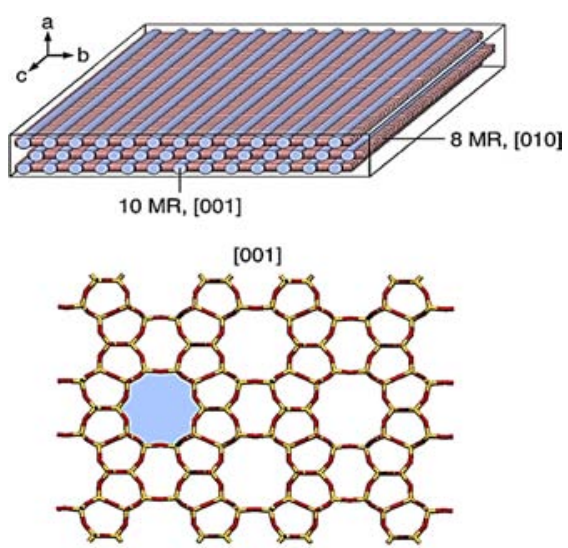

[010]

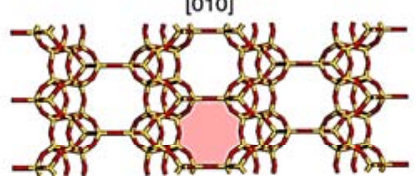

P

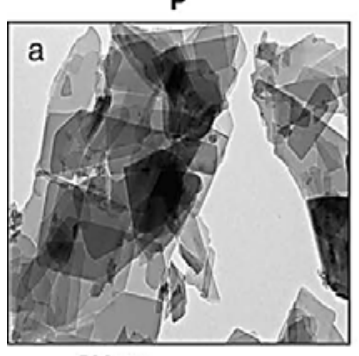

$-500 \mathrm{~nm}$

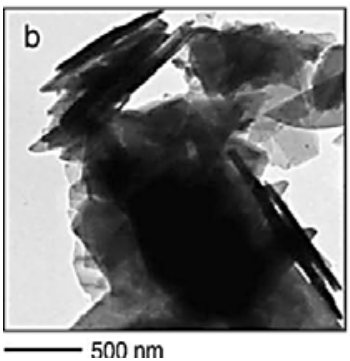

AT-16

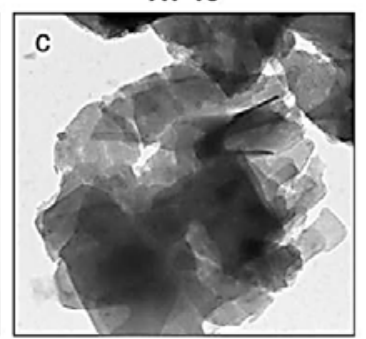

$-500 \mathrm{~nm}$

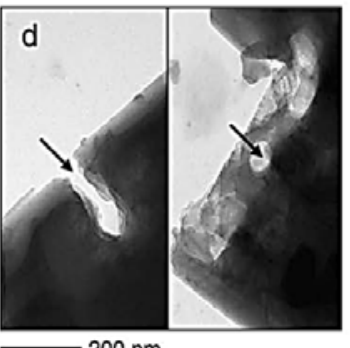

$200 \mathrm{~nm}$
AT-11
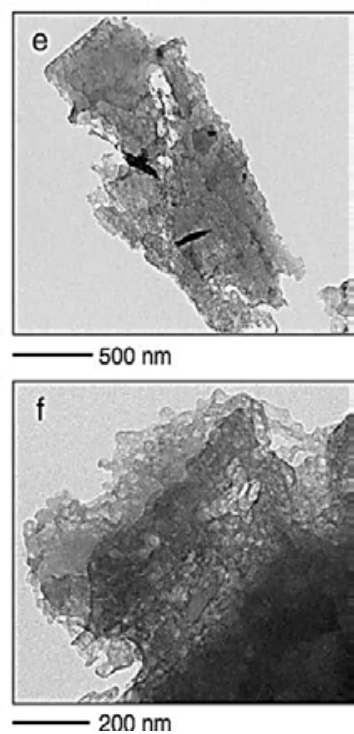

Fig. 9. Structure of ferrierite and its 10-MR and 8-MR pores (colored parts). TEM images of parent ferrierite (P) and alkali-treated samples (AT-16 and AT-11, obtained using 0.2 and $0.5 \mathrm{~mol} / \mathrm{L} \mathrm{NaOH}$, respectively). (a, b) Single platelets of parent ferrierite of length $0.3-0.8 \mu \mathrm{m}$ and lateral width 50-150 nm; (c) Roughness of crystal external surface with respect to parent sample, caused by base attack; (d) Fissure formation at crystal edge, shown by arrows; (e, f) More-damaged ferrierite platelets and formation of holes (mostly perpendicular to crystal planes), caused by harsher desilication conditions compared with those for AT-16. Reprinted with permission from Ref. [98]. Copyright 2009, Elsevier. 


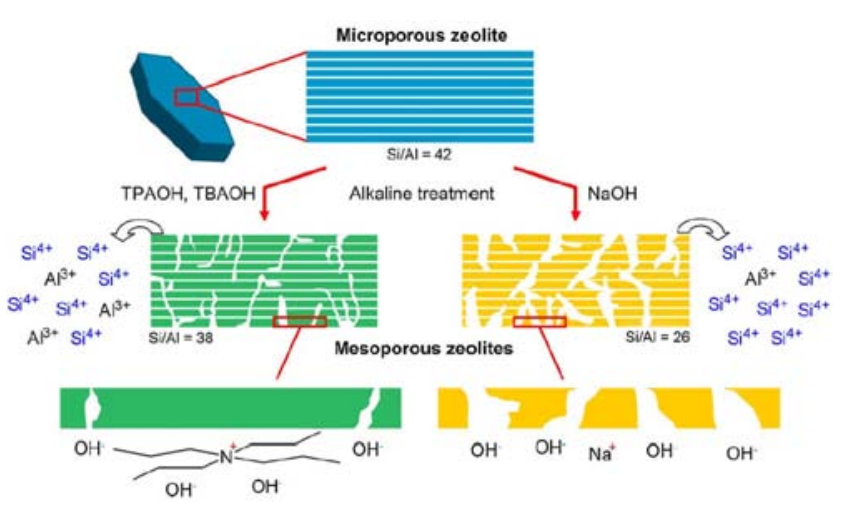

Fig. 10. Comparison of zeolite desilications using $\mathrm{NaOH}$ and tetraalkylammonium hydroxides. Reprinted with permission from Ref. [101]. Copyright 2009, Elsevier.

\subsubsection{Synthesis of hierarchical zeolites by surfactant-assisted recrystallization method}

Mesoporosity can be effectively introduced into zeolitic structure using mild conditions (e.g., dilute $\mathrm{NaOH}$ solution, low temperature and short time) together with a cationic surfactant such as cetyltrimethylammonium bromide or chloride (CTAB or CTAC), i.e., recrystallization of microporous zeolites to micro-mesoporous ones or meso-structuring of zeolites. This process prevents dissolution of the crystals by providing interactions between the surfactant and the zeolite, and almost complete recovery is achieved as a result of reorganization of the zeolite lattice around surfactant micelles [102-104]. Ivanova et al. [105] used several techniques, including X-ray diffraction (XRD), multinuclear MAS NMR, and thermogravimetric analysis, to investigate the mechanism of a single-step recrystallization process at the molecular level by considering the intermediate products. MOR was used as a typical zeolite and $\mathrm{NaOH}$ basic solution containing CTAB surfactant was used to hydrolyze and recrystallize the sample during hydrothermal treatment (the proposed mechanism is shown in Fig. 11). They showed that after initial breakage of $\mathrm{Si}-\mathrm{O}-\mathrm{Si}$ bonds in the alkaline medium, large inter- and intra-crystalline pores were formed during desilication. CTAB species then diffused into these spaces, CTA cations were exchanged with framework $\mathrm{Na}$, and micelles formed. Finally, agglomeration of siliceous species surrounding the micelles gave a single-phase zeolite with a bimodal porous structure. In another study, Na et al. [106] mixed a certain mass of HZSM-5 zeolite with a typical $\mathrm{NaOH}$ aqueous solution and added an aqueous solution of alkyltrimethylammonium bromide (with various chain lengths to manipulate the mesopore sizes) to the suspension, followed by hydrothermal treatment. Composites of ZSM-5 and MCM-41 were formed using this two-step desilication-recrystallization method. The zeolite recrystallization procedure has been discussed comprehensively in an extensive review [107]. In terms of the degree of zeolite recrystallization, various micro-mesoporous materials involving mesostructured zeolite crystallites (the crystal surfaces may be covered with mesoporous materials), and two coexisting crystalline zeolitic and mesoporous substances forming composites, and mesoporous

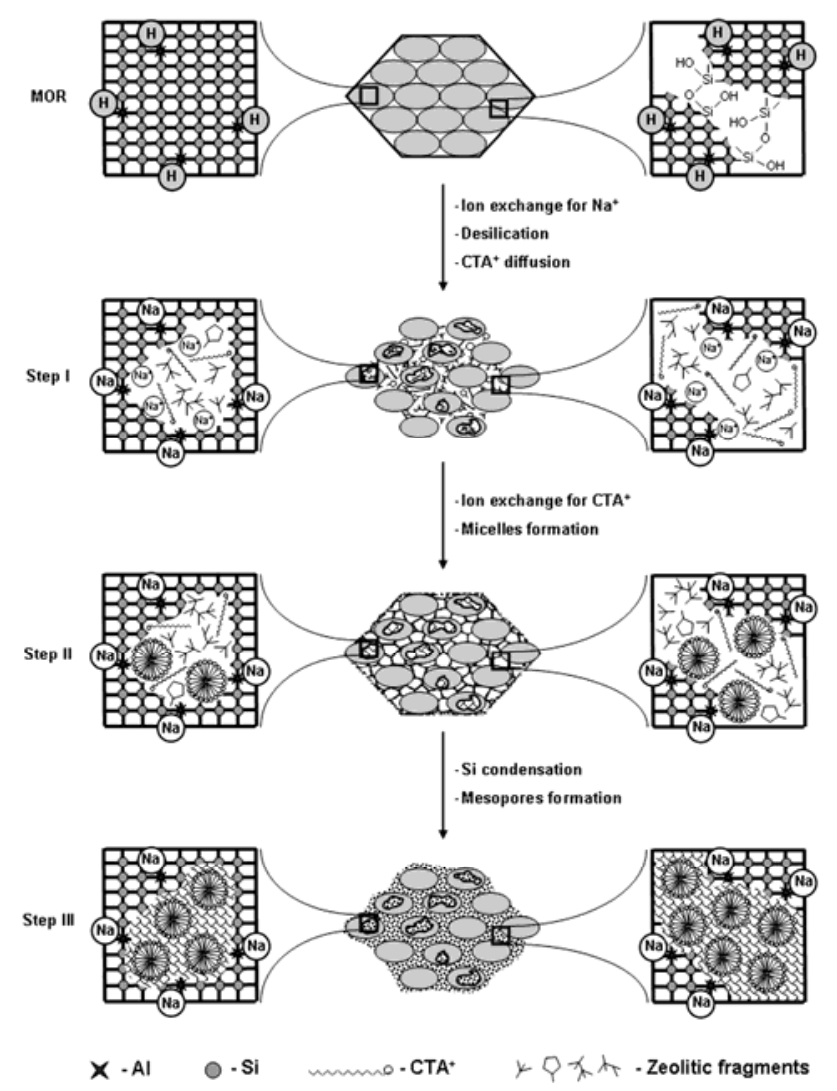

Fig. 11. Mechanism of mordenite desilication/recrystallization to micro-mesoporous structure. Reprinted with permission from Ref. [105]. Copyright 2014, Elsevier.

materials with small zeolitic parts in their walls can be obtained.

To summarize, the preparation of hierarchical zeolites using surfactant-assisted recrystallization is an easy industrial process that prevents the framework damage that occurs in the desilication method.

\subsubsection{Synthesis of hierarchical zeolites by zeolitization of mesoporous/macroporous starting materials}

Extensive studies have enabled the conversion of mesoporous structures with amorphous walls to crystalline zeolite frameworks. Dry gel conversion in the presence of an organic microporous SDA resulted in ordered mesoporous substances such as SBA-15 and MCM-41 undergoing partial crystallization on their walls to give a zeolitic structure $[108,109]$. This method leads to intercrystalline mesopore formation. Clearly, the morphology, topology, and phase purity of the zeolite crystals, and the distribution, location, size, shape, and interconnectivity of the mesopores, are closely related to the template type and how well they interact with aluminosilicate species in the synthesis gels $[23,102]$. Unfortunately, during conventional hydrothermal synthesis, the zeolite and mesoporous substrates nucleate synchronously, resulting in the formation of a physical mixture consisting of both components. Moreover, crystallization of mesopore walls to zeolites to form the resultant product, which have different crystalline and amorphous phase densities, causes breakage of the aluminosilicate framework, so 
partial collapse of the final meso-structured material occurs [110].

In another approach, careful deposition of presynthesized nanozeolite seeds onto an ordered/non-ordered macro- or meso-porous support resulted in zeolitized porous materials [111]. Wang et al. [112] prepared hierarchical zeolites using a vapor-phase transport (VPT) method to transform macroporous diatomites into zeolites in the presence of zeolite seeds. First, they synthesized nanosized crystals of silicalite-1 and $\beta$ zeolites of average sizes 80 and $45 \mathrm{~nm}$, respectively, through hydrothermal synthesis. Then, layer-by-layer electrostatic deposition was used to cover them with diatomites. Diatomite modification was first performed by the deposition of one layer of a cationic polyelectrolyte, i.e., poly(diallyldimethylammonium chloride) (PDDA, $M_{\mathrm{r}}<200000$ ), followed by several cycles in which nanozeolite seeds and PDDA were periodically deposited onto the pretreated diatomites until uniform zeolite/PDDA multilayers were formed. Next, the nanozeolite-seeded diatomites underwent VPT treatment to obtain zeolitized diatomites. The results show that colloidal zeolite seeds induced zeolitization of diatomites, and diatomaceous silica without seeds was not active enough to be directly transformed into zeolites. In another study, diatomite zeolitization was performed with the aid of silicalite- 1 nanocrystals and CTAB surfactant and steam-assisted treatment [111]. The role of CTAB was to restrain the size of the zeolite crystals formed on the diatomite surface. During crystallization induced by steaming at $150{ }^{\circ} \mathrm{C}$ for $72 \mathrm{~h}$, the zeolite microstructure changed, as shown by the XRD patterns and $\mathrm{N}_{2}$ adsorption-desorption isotherms (Fig. 12). The intensities of the reflection peaks are comparable to those of the MFI framework, and the micropore volume and BET surface area of the final zeolitized diatomite increased with increasing nanoprecursor quantity. All zeolitized diatomite samples had micropore sizes in the range 0.55-0.56 nm, corresponding to the channel opening size of silicalite-1, and mesopores (approximately $4 \mathrm{~nm}$ ) related to close-packed silicalite-1 nanocrystals. Scanning electron microscopy (SEM) images of the samples showed uniform growth of silicalite- 1 nanocrystals (ca. $300 \mathrm{~nm}$ in size) and a preserved hierarchical porous structure, as well as cylindrical zeolites. Larger zeolite crystals were obtained by using ethanol instead of water as the dispersion medium, because the dielectric constant of ethanol is lower than that of water.

The method used to introduce nanozeolite seeds into mesoporous structures is clearly an important subject. Better yields are obtained by wet impregnation from a clear solution rather than mechanical mixing of both powders before calcination. However, zeolite particle deposition on the external surfaces of the porous supports can cause pore entrance blockage [23].

\subsubsection{Synthesis of hierarchical zeolites by nanoparticle assembly}

Mesopores in a hierarchical zeolite can be present in three forms: inter-crystalline, intra-crystalline, and both between inside crystals. A hierarchical zeolite with an intercrystalline mesoporous structure can be obtained by controlling the conditions to reinforce nucleation, nanozeolite preparation, and aggregation of nanozeolite seeds with voids between them (Fig. 13). These materials give better performances than conventional zeolites because of two good features, i.e., the nanoscale zeolite crystals have much higher external surface areas and the presence of larger pores among the nanocrystals acceler-
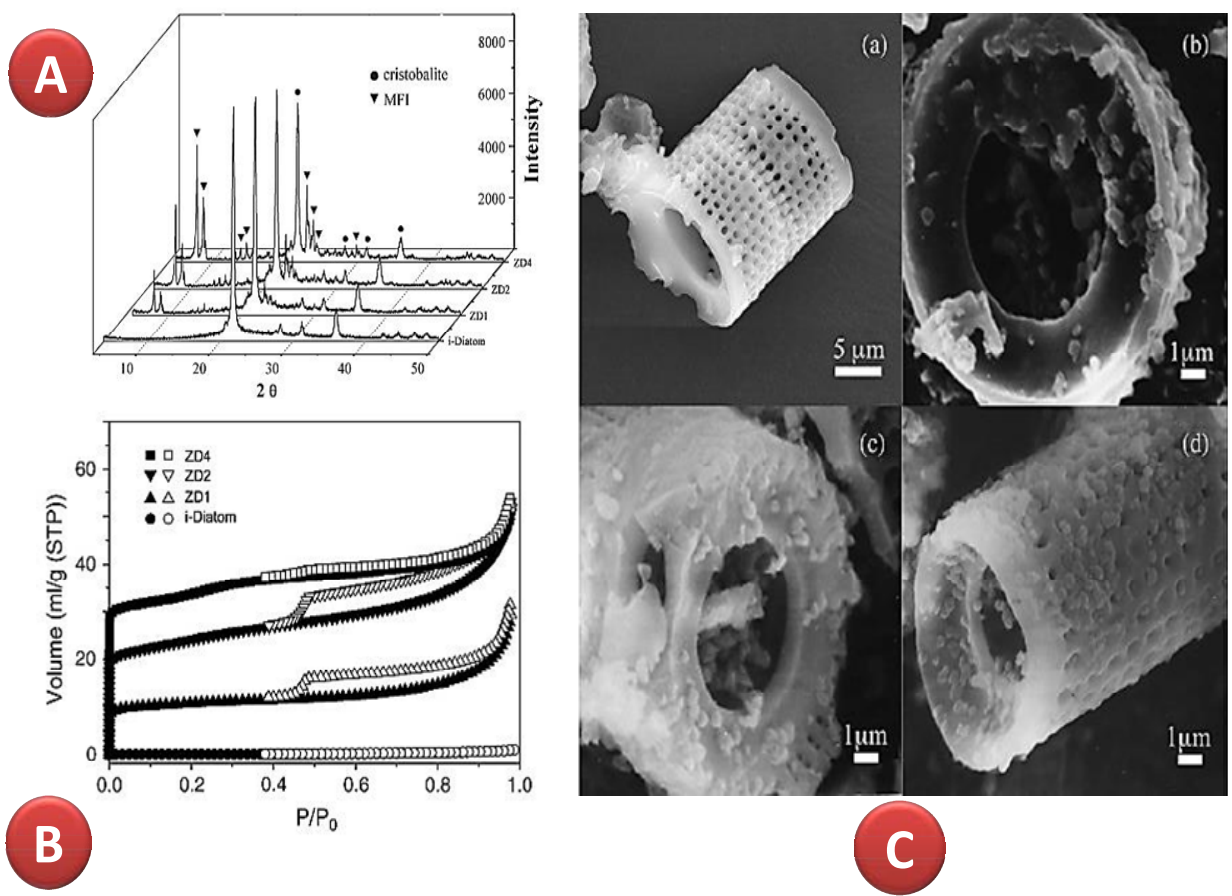

Fig. 12. (A) XRD patterns and (B) $\mathrm{N}_{2}$ adsorption-desorption isotherms of initial diatomite (i-Diatom) and zeolitized diatomites [ZD $x, x=$ $10\left(W_{\text {diatom }} / W_{\text {silicalite-1 }}\right), W_{\text {diatom }}$ and $W_{\text {silicalite-1 }}$ are added weights of silica in the diatomite and silicalite-1 precursors, respectively], and (C) SEM images of (a) i-Diatom, (b) ZD1, (c) ZD2, and (d) ZD4. Reprinted with permission from Ref. [111]. Copyright 2008, Elsevier. 
Nanozeolite Aggregates

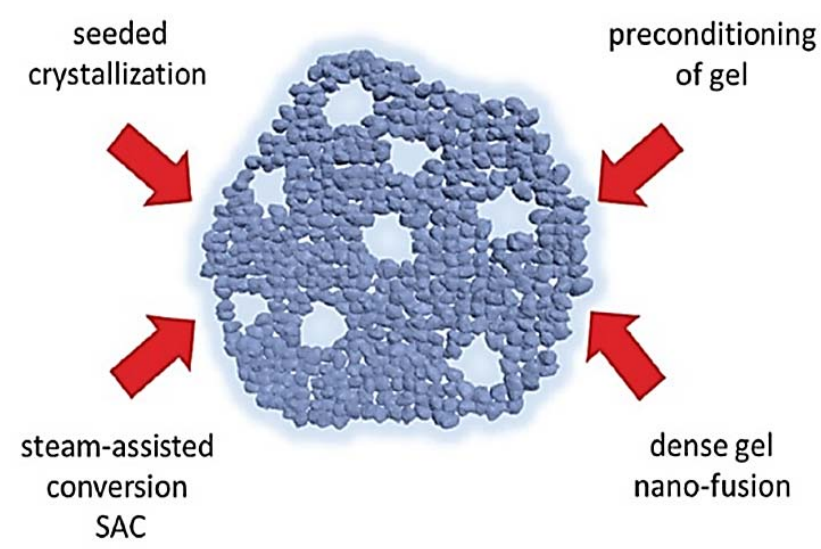

Fig. 13. Assembly of nanozeolites to form mesoporous zeolites. Reprinted with permission from Ref. [91]. Copyright 2013, Royal Society of Chemistry.

ates mass transport of the reactants and products [113].

There is connectivity between the micropores and meso- or macro-pores throughout the hierarchical zeolite, therefore molecules can be effectively transported through the ideal pore architecture. Zeolite nanocrystals can self-assemble to give hierarchical structures through various routes such as sorption or growth onto three-dimensional supports such as porous polymer beads, by gathering around surfactant micelles and polymers, dry gel conversion, in which a dry gel is crystallized at high temperature in a steam flow of SDA/water, and steam-assisted conversion (SAC), in which a crystalline hierar- chical zeolite is obtained via crystallization of a dry gel containing an organic template under water-steaming treatment [114]. Another approach to mesoporous zeolite synthesis is the use of specifically designed amphiphilic organosilanes as mesoporogens as well as micropore templates. Serrano and coworkers [115] studied the synthesis of a nanocrystalline ZSM-5 zeolite with hierarchical porosity. Zeolite seeds were prepared under reflux, and then phenylaminopropyltrimethoxysilane (PHAPTMS) was used as a silanizing agent to functionalize the as-prepared seeds (Fig. 14).

The authors reported that the adopted route involved four steps: precrystallization of the starting synthesis gel containing TPAOH, which is a common SDA, to produce zeolite seeds with the MFI framework; silanization through functionalization of the external surfaces of zeolitic nanoparticles using an organosilane substrate; supplementary zeolitization by crystallization of the functionalized seeds; and removal of TPAOH and PHAPTMS species via calcination at high temperature in air. The organosilane agent inhibited nanoparticle aggregation, enabling formation of large crystals. The results confirmed the appearance of new interstitial voids between the nanocrystals in the mesopore range, and it was found that without the precrystallization stage, synthesis of a nanozeolitic structure with hierarchical porosity was not possible, because of imperfect crystallization of ZSM-5 zeolite. However, different results have been reported by others [113]. Möller et al. [116] investigated the preparation of hierarchical $\beta$ zeolite by assembling nanoparticles in the presence of PDDA as a secondary SDA in the primary dense synthesized gel. In the absence of the cationic polymer, only colloidal nanoparticles of $\beta$ zeolite were obtained (the individual particle sizes and yield of $\beta$ zeolite were $40-130$

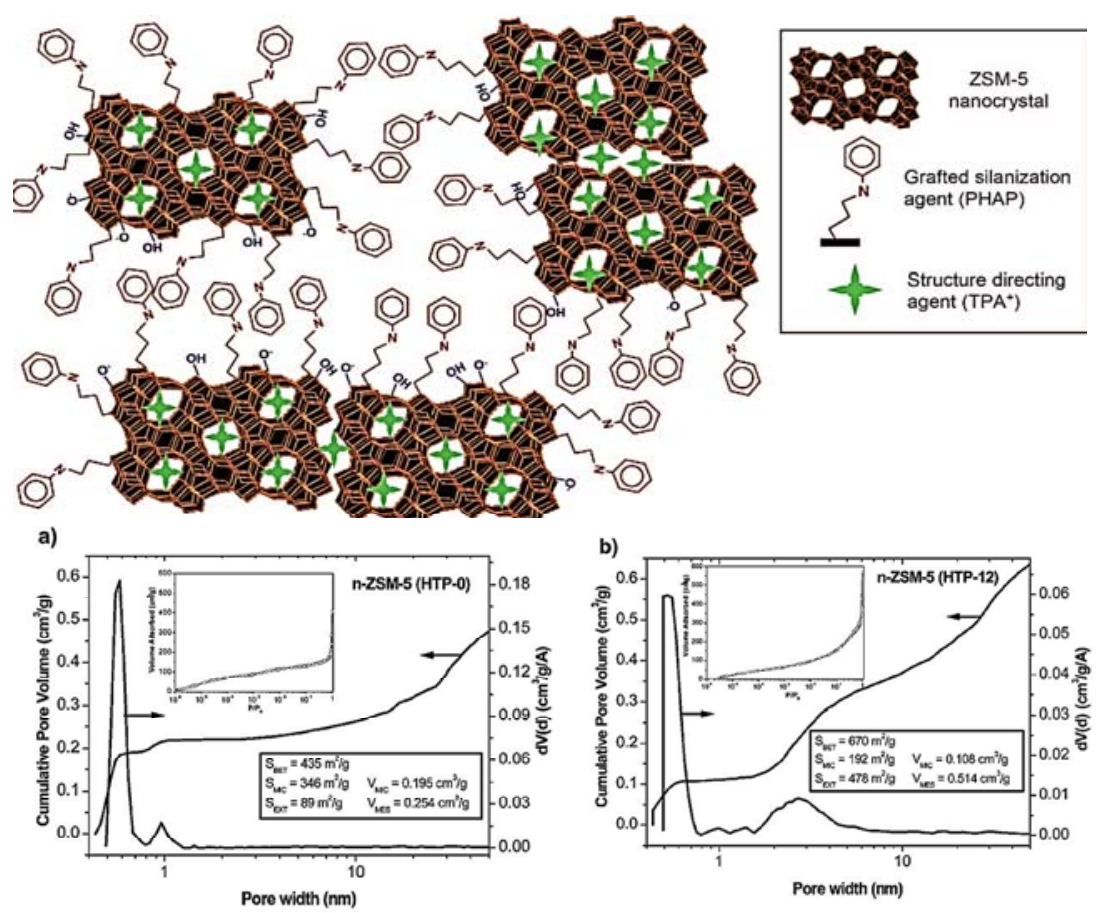

Fig. 14. Schematic diagram of PHAPTMS surface-silanizing and $\mathrm{TPA}^{+}$zeolite-structure-directing species distributions with respect to exterior surfaces and interior nanopores of ZSM-5 zeolite nanocrystals (top). Pore size distributions for calcined nano-ZSM-5 zeolites prepared under high-temperature precrystallization $\left(90^{\circ} \mathrm{C}\right.$ ) conditions with (a) $0 \%$ and (b) $12 \%$ PHAPTMS (bottom). Reprinted with permission from Ref. [115]. Copyright 2009, American Chemical Society. 
$\mathrm{nm}$ and almost 75\%, respectively). The presence of PDDA induced nanoparticle flocculation and formation of meso/macropores as interparticle voids, the size of which could be tuned based on the amount of polymer (Fig. 15). These results show that PDDA addition increased the mesopore/macropore size, but the crystallinity of the end product was lower because the larger polymer content prevented efficient conversion of the amorphous gel. The molecular mass of the polymer affected the process to some extent. Fang and coworkers [117] attempted to produce mesoporous aggregates of zeolite ZSM-12 via in situ assembly of nanoparticles from a concentrated synthesized gel that did not contain a mesoporogen. They used supersaturated conditions, therefore zeolite nucleation prevailed over growth. First, conventional zeolite ZSM-12 was prepared. The mesoporous zeolite was prepared using the same gel as was used to make the conventional ZSM-12 zeolite, but which had been concentrated by refluxing to get rid of water and alcohol and reduce the $\mathrm{H}_{2} \mathrm{O} / \mathrm{SiO}_{2}$ molar ratio. Polycrystalline particles consisting of primary nanozeolites were obtained by self-assembly of unstable individual nanocrystals under hydrothermal conditions. Finally, intercrystalline mesoporosity arose from incomplete intergrowth of zeolitic nanocrystals. The discussed methods generally achieve a high degree of conversion with high crystalline yields, minimizing waste and reducing the consumption of expensive organic templates.

\subsubsection{Template-assisted synthesis of hierarchical zeolites}

The use of soft or hard meso templates in addition to microporous SDAs in conventional zeolite synthesis gels can induce additional porous systems in the zeolite framework structure. There have been many studies of the use of hard
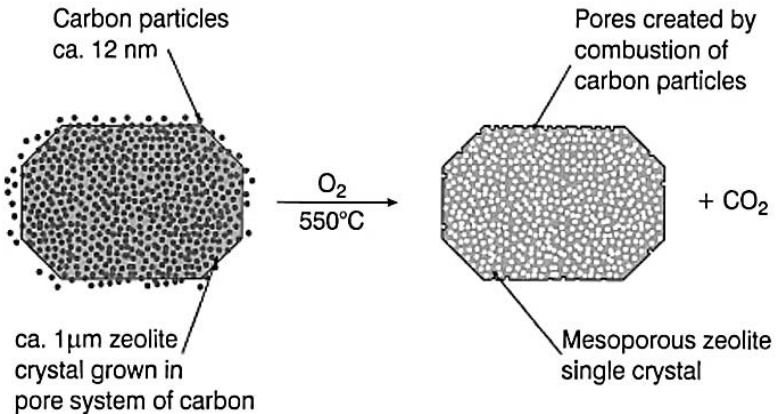

Fig. 16. Nucleation and growth of zeolite crystals around carbon particles and within pores. Reprinted with permission from Ref. [118]. Copyright 2000, American Chemical Society.

templates with zeolite synthesis gels. In the preparation of ZSM-5 nanozeolites using a confined-space method, an initial product is formed by excess gel adsorption on carbon black particles at the impregnation stage (Fig. 16). After zeolite crystal formation, combustion in air is conducted to remove the carbon template and obtain mesopores comparable to the carbon particle size [118].

Other carbon sources such as carbon nanotubes, carbon aerogels, and sucrose have also been used as hard templates [21,119-122]. There are two types of defect associated with carbon templates. The first is phase separation between hydrophobic carbon and the hydrophilic synthesis gel, which can be rectified by using steam in the reaction system (SAC). The other is high-temperature combustion of carbon compounds, which results in loss of product, and high costs; these factors should be taken into account in industrial applications [21,91]. Zhu et al. [123] used nanosized $\mathrm{CaCO}_{3}$ as a hard template to
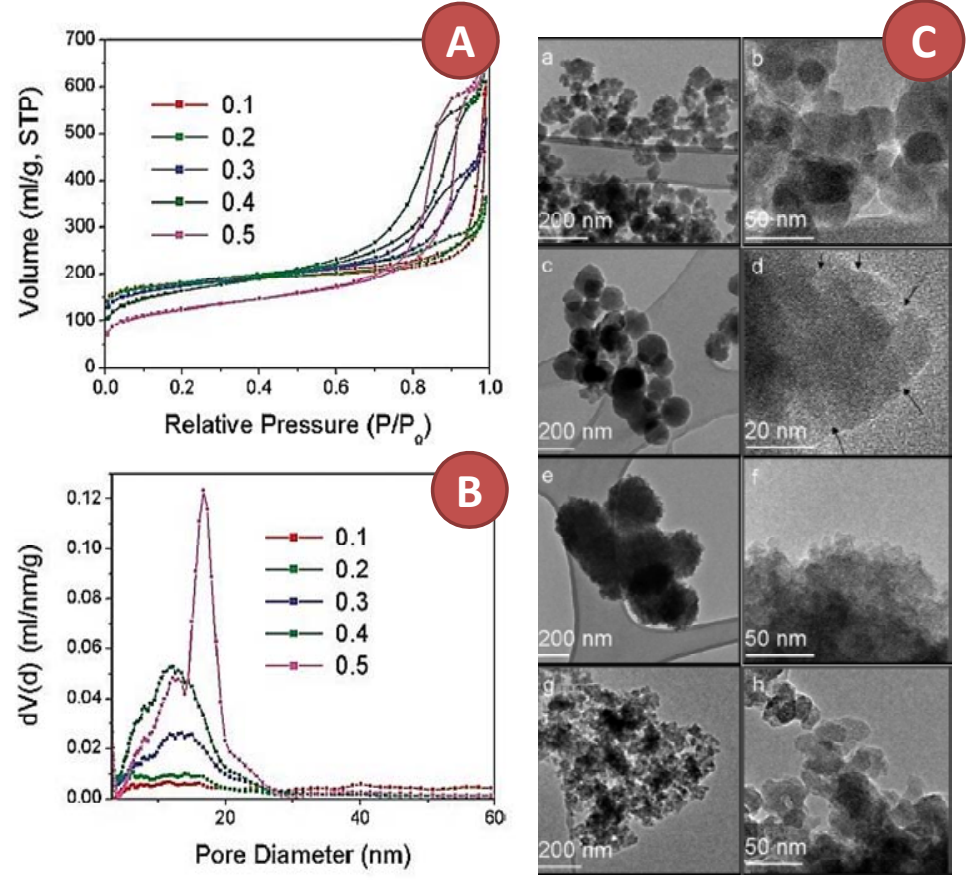

Fig. 15. Effects of increasing amount of low-molecular-mass PDDA on (A) nitrogen sorption isotherms and (B) pore-size distributions of $\beta$ zeolites. (C) TEM images of zeolite samples with addition of (a, b) no polymer, (c, d) 0.1 g PDDA/g SiO 2 , (e, f) 0.2 g PDDA/g SiO , and (g, h) 0.3 g PDDA/g SiO 2 . Reprinted with permission from Ref. [116]. Copyright 2011, American Chemical Society. 
synthesize silicalite- 1 zeolite. The template nanoparticles were removed by acid treatment and auxiliary mesopores with nanosized-carbonate particle diameters remained. The results show that only interactions between hydrophilic $\mathrm{CaCO}_{3}$ with surface hydroxyl groups and $\mathrm{SiO}_{2}$ species in the silica gel promoted hierarchical zeolite formation, because hydrophobic $\mathrm{CaCO}_{3}$, which was produced by fatty acid modification of a hydrophilic carbonate, did not affect secondary pore system formation and only the traditional silicalite- 1 structure was obtained. The preparation of hierarchical ZSM-5 was also performed using bread (a polymeric starch matrix) as a hydroxyl-group-rich mesoporogen [124]. In the synthesis, bread was added to the initial clear solution of precursors. The mixture was transferred to an autoclave for hydrothermal conversion. The product was a hierarchical zeolite with intracrystalline mesopores. The authors studied the hydrothermal stability of the final product by exposing it to boiling water. A small decrease in the BET surface area of mesoporous ZSM-5 was observed; when mesoporous silica SBA-15 was subjected to boiling water for shorter times, a 72\% reduction in the BET surface area was observed. This shows that the stability of meso-ZSM-5 is higher than that of meso-SBA-15.

The use of conventional tetraalkylammonium cations and large templates as SDAs for the formation of micropores and mesopores, respectively, is an easy method for producing bimodal zeolites. Various polymers, copolymers, organosilanes, and surfactants, as well as carbons, can act as flexible soft templates [125-127]. Various factors determine the suitability of mesoporogen for the synthesis of a mesoporous zeolite. The hydrothermal stability of the meso template under the reaction conditions is an important issue because it must not be damaged during the process. Appropriate interactions between silica species and the template is another crucial factor; positively charged templates are preferred because silica species are negatively charged in the alkaline solutions that are usually used in the synthesis. It should also be noted that the shape of the imported mesopores depends on the template morphology [128]. Tao et al. studied the preparation of a hierarchical ZSM-5 zeolite with intracrystalline mesopores and intercrystalline macropores using SAC with poly(ethylene glycol) (PEG) as a meso template [129].

First, they produced a dry gel containing silica, alumina, $\mathrm{PEG}$, and a high ratio of $\mathrm{TPA}^{+}$to $\mathrm{SiO}_{2}$. The dry gel was converted to zeolite nanocrystals. They reported that the origin of this phenomenon was the high concentration of a micropore SDA, which promoted nucleation. These crystals encapsulated the PEG in the dry synthesis gel until intracrystalline mesopores were introduced after its removal. Nano-unit aggregation led to intercrystalline macropore formation during the steam-assisted treatment (Fig. 17). Natural macromolecular polymers such as starch and sodium carboxymethylcellulose have been used as mesoporogens in the synthesis of MFI zeolites [130]. These substances interact with $\mathrm{Si}-\mathrm{OH}$ in the zeolite gel via hydrogen bonding, because of the abundant hydroxyl groups on their long chains. They were directly added to the synthetic medium and the mesoporosity remained after calcination. The amounts of alkali and water, aging temperature,

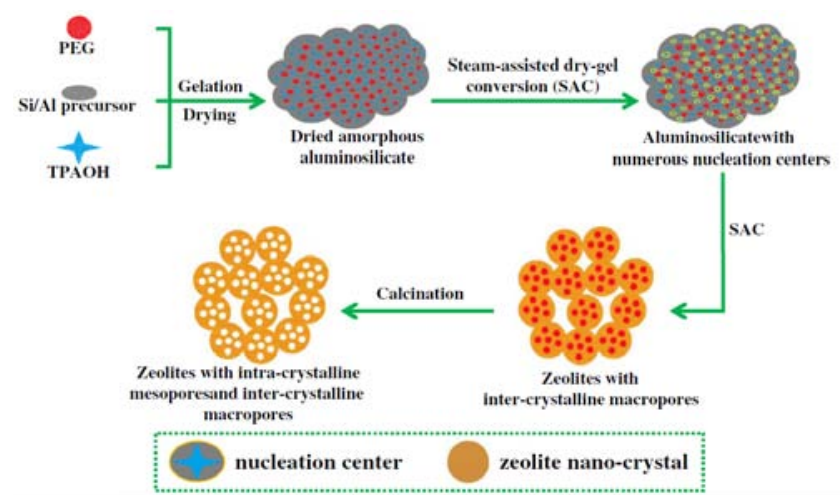

Fig. 17. Formation of intra- and inter-crystalline porous systems in hierarchical ZSM-5 zeolite via SAC in the presence of PEG. Reprinted with permission from Ref. [129]. Copyright 2013, Elsevier.

and template affected the mesoporous structure and phase composition. Extra alkali and less water led to hybrid phase formation; mesopores were not formed in the presence of a large amount of water, and a lower alkali concentration reduced the zeolite crystallinity. The template viscosity and $\mathrm{pH}$ also affected the reaction. In a recent study, researchers used only one non-surfactant cationic polymer, namely PDDA, to induce both micro- and meso-pore formation in the $\beta$ zeolite structure [131]. The high density of quaternary ammonium groups on the polymer resulted in conventional micropore formation and agglomeration of the flexible macromolecules in the zeolite synthesis system during crystallization produced mesoporous structures. Incorporation of the polymer into the mesoporous $\beta$ zeolite during crystallization was observed in the ${ }^{13} \mathrm{C}$ NMR spectrum of the solid product as broad and weak peaks, because of the low polymer content (Fig. 18); the polymer remained unscathed in the prepared mesoporous zeolite. Thermogravimetric analysis showed a mass loss of nearly $28 \%$ in the temperature range $250-550{ }^{\circ} \mathrm{C}$; this is attributed to polymer decomposition. As mentioned above, soft templates
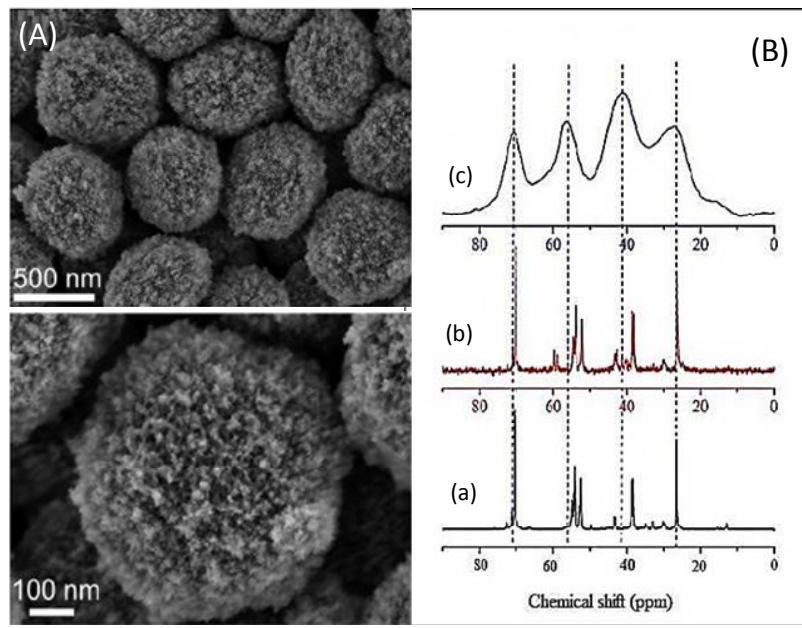

Fig. 18. (A) SEM images of calcined mesoporous $\beta$ zeolite at different magnifications. (B) ${ }^{13} \mathrm{C}$ NMR spectra of (a) aqueous PDDA, (b) liquid phase obtained by dissolving as-synthesized mesoporous $\beta$ zeolite in $\mathrm{HF}$ solution, followed by addition of $\mathrm{D}_{2} \mathrm{O}$, and (c) solid sample of as-synthesized mesoporous $\beta$ zeolite. Reprinted with permission from Ref. [131]. Copyright 2014, American Chemical Society. 
produce intracrystalline mesopores, and intercrystalline mesopores are formed by nanozeolite aggregation.

\subsection{Catalytic applications of hierarchical zeolites}

Hierarchical zeolites have potential applications in many fields because of their properties, and various methods for their synthesis have been developed. For reactions involving the diffusion of small molecules into micropores, the results achieved using conventional and hierarchical zeolites are similar; however, reactions involving bulky substrates profit from the multimodal porosities of hierarchical zeolites [132]. Alkylation, isomerization, methanol to hydrocarbons (MTH) conversion, aromatization, polymer pyrolysis, condensation, and catalytic cracking reactions are among the many types of reactions that benefit from the use of hierarchical zeolites $[94,98,101,103,104,106,113,115]$. For example, in the alkylations of benzene with isopropanol using conventional and hierarchical $\beta$ zeolites, higher activity and selectivity for the main product (isopropylbenzene) were achieved in the presence of mesoporous zeolites, as a result of improved mass transfer and the secondary pore system [133]. Similar results were obtained for catalytic cracking of 1,3,5-triisopropylbenzene using a hierarchical ZSM-5 zeolite prepared from a synthesis gel containing poly(ethylene oxide) as a meso template [134]. As mentioned earlier, one important problem that microporous structures usually face during catalytic processes is blockage of the pore mouths as a result of coke deposition, resulting in catalyst deactivation. Coke formation is unavoidable. It poisons the catalyst and cannot be recovered, and therefore it is essential to minimize coke formation [28]. Many studies have shown that coke formation can be avoided using the large pore systems in mesoporous zeolites [104,106,127,135]. In most cases, improved catalytic performances, e.g., enhanced catalytic conversions, improved selectivities, restricted coking and catalyst deactivation, and longer catalyst lifetimes, are observed because of the auxiliary mesoporosity in the zeolite systems, which enables these materials to be used as efficient catalysts in a wide range of reactions [86]. Readers are referred to the detailed review on catalytic reactions involving mesoporous zeolites by Holm and coworkers [86]. They investigated the performances of hierarchical zeolites in various reactions such as cracking, alkylation, isomerization, condensation, olefin aromatization, and MTH conversion; the reported roles of mesoporous zeolites in different catalytic reactions are shown in Fig. 19.

Holm et al. asserted that it was possible to predict the required mesoporosity level for each reaction by comparing the properties of numerous hierarchically structured zeolites. Additionally, it is crucial to consider the reaction site, i.e., whether reactions take place only on the mesopore/external surfaces, at the pore entrances, or inside the micropores.

\subsection{Comparison of catalytic applications of nanosized and hierarchical zeolites}

Many studies have confirmed the success of mesoporosity

\begin{tabular}{|ll|}
\hline Reaction type & Main observation \\
\hline Alkylation reactions & Higher activity \\
Transalkylation reactions & Improved stability \\
Isomerisation reactions & Higher activity \\
Cracking of light substrates & No significant improvement \\
Cracking of heavy substrates & Substantially higher activity \\
MTH and aromatization reactions & Longer catalyst lifetime \\
Condensation reactions & Higher activity. Higher selectivity \\
& towards bulky products \\
\hline
\end{tabular}

Fig. 19. Effects of mesoporous zeolites in different catalytic reactions. Reprinted with permission from Ref. [86]. Copyright 2011, Elsevier.

introduction into zeolite frameworks or reduction in their crystal size in developing catalysts for a wide range of reactions. It is informative to compare the performances of the nanocrystalline and hierarchical versions of a conventional zeolite in a specific reaction. Rownaghi et al. [136] recently reported differences between the catalytic efficiencies of nanoand meso-ZSM-5 zeolites in the dehydration of methanol to dimethyl ether (DME) (Fig. 20). The reactions were performed in a fixed-bed reactor with continuous flow at temperatures from 180 to $320^{\circ} \mathrm{C}$. In all experiments, increasing the temperature enhanced the reactivity, and the conversions achieved using nanocrystalline and mesoporous zeolites were significantly higher than those obtained using conventional large micropore zeolite crystals (nano-ZSM-5 > meso-ZSM-5 >> con-ZSM-5). This is the result of improved diffusion into channels and access to acid sites for the reactant molecules. The conversion of methanol to hydrocarbons involves various reactions, i.e., dehydration to water and DME and conversion of DME to light olefins. An increase in the reaction temperature when using a conventional zeolite as the catalyst decreased the selectivity for DME over hydrocarbons. Increasing the temperature from 180 to $225^{\circ} \mathrm{C}$ did not change the DME selectivity for either the nano- or meso-ZSM-5 zeolite, but the conversion and DME selectivity at $270{ }^{\circ} \mathrm{C}$ were higher with nano-ZSM-5 than with meso-ZSM-5. From 270 to $320^{\circ} \mathrm{C}$, the hydrocarbon selectivity (C1-C10) increased to $98.5 \%$ (total selectivity was achieved at $320{ }^{\circ} \mathrm{C}$ ). The on-surface to in-crystal ratio, $\mathrm{Al} /(\mathrm{Si}+\mathrm{Al})(\mathrm{AS} / \mathrm{AC})$, and the crystal size are responsible for the DME selectivity, which decreases from $100 \%$ to zero as AS/AC decreases. The differences among the $\mathrm{SiO}_{2} / \mathrm{Al}_{2} \mathrm{O}_{3}$ ratios of the three catalysts lead to differences among their surface properties and different conversions and product distributions. The highest DME selectivity was achieved using nano-ZSM-5 zeolite (ca. $48 \%$ at $270{ }^{\circ} \mathrm{C}$ ). This confirms the importance of the pore channel dimension of the nanozeolite for DME selectivity, as it limits hydrocarbon production during further dehydration reactions.

Three different types of MFI zeolite (conventional, nanosized, and mesoporous zeolites) were prepared from one synthetic mixture containing a single organic template by altering the conditions. Their activities in the catalytic conversion of methanol to gasoline-range hydrocarbons [137] were investigated. The nanocrystalline and mesoporous zeolites both had high catalytic stability and product selectivity. The best results for methanol dehydration and the highest selectivity for light 


\begin{tabular}{|c|c|c|c|c|c|c|c|c|c|c|}
\hline \multirow{2}{*}{ Catalyst } & \multirow{2}{*}{$\begin{array}{c}\text { Crystal size } \\
(\mu \mathrm{m})\end{array}$} & \multirow{2}{*}{$\begin{array}{c}S_{\mathrm{BET}} \\
\left(\mathrm{m}^{2} \mathrm{~g}^{-1}\right)\end{array}$} & \multirow{2}{*}{$\begin{array}{l}S_{\text {External }} \\
\left(\mathrm{m}^{2} \mathrm{~g}^{-1}\right)\end{array}$} & \multicolumn{2}{|c|}{$\mathrm{Al} /(\mathrm{Si}+\mathrm{Al})(\mathrm{mol} \%)$} & \multirow{2}{*}{$\mathrm{AS} / \mathrm{AC}$ ratio } & \multicolumn{4}{|c|}{ Number of acid sites $\left(\mathrm{mmol} \mathrm{NH}_{3} / \mathrm{g}\right)$} \\
\hline & & & & In crystal (AC) & On surface (AS) & & Weak & Moderate & Strong & Total \\
\hline Nano-ZSM-5 & 0.12 & 379 & 113 & 3.48 & 3.58 & 1.03 & - & - & 27.00 & 27 \\
\hline Meso-ZSM-5 & 0.30 & 370 & 138 & 3.45 & 3.76 & 1.09 & - & 0.10 & 0.18 & 28 \\
\hline
\end{tabular}
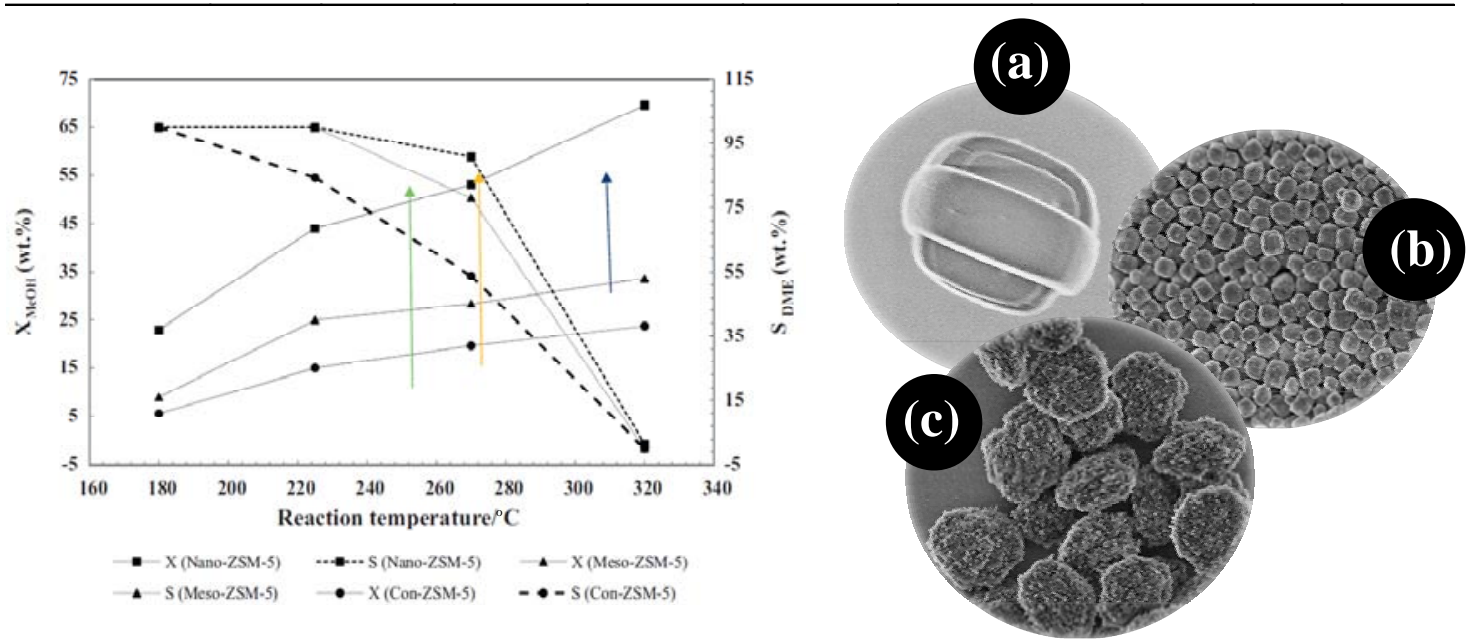

Fig. 20. SEM images of ZSM-5 crystals synthesized via different methods and their physical and chemical properties. (a) Con-ZSM-5; (b) Nano-ZSM-5; (c) Meso-ZSM-5. Curves show changes in methanol conversion and DME selectivity with reaction temperature for protonic form of catalysts. Reprinted with permission from Ref. [136]. Copyright 2012, Elsevier.

olefins were obtained using uniform ZSM-5 nanocrystals, because of their higher surface area and larger number of acid sites on the external surface of the catalyst. In contrast, higher yields of paraffins and alkyl aromatic selectivity were obtained with meso-ZSM-5, possibly because of the channel structure of crystals with secondary porosity. It seems that for a precise comparison of the performances of nanozeolites and mesoporous zeolites, to enable decisions to be made on which form to use, the experimental conditions should be identical for both structures. In a recent study, Yan et al. [138] investigated the catalytic applications of hierarchical and nanosized zeolites in liquid and gaseous reactions (Fig. 21).

For reactions occurring inside the micropores (e.g., catalytic conversion of methanol to hydrocarbons) the product selectivity is improved by the shorter micropore channels and larger number of weak acid sites provided by nanosized zeolites. In

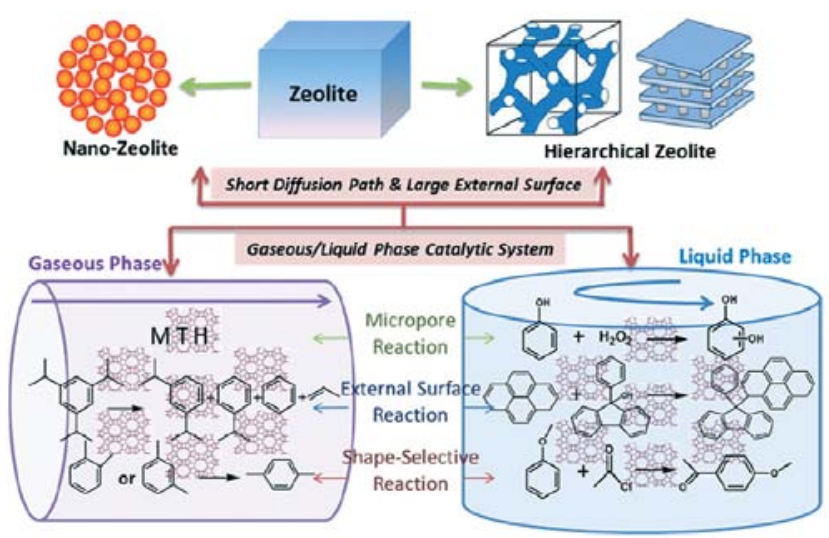

Fig. 21. Catalytic applications of hierarchical and nanosized zeolites in liquid- and gas-phase reactions. Reprinted with permission from Ref. [138]. Copyright 2015, Royal Society of Chemistry. many cases, because of the large surface area, the rate of deactivation and lifetimes are shorter and longer, respectively. The mesopore properties and framework defects also affect the rate of catalyst deactivation. For reactions that tend to occur on the external surfaces of catalysts, strong acidity and larger numbers of available active sites lead to better cracking conversion of bulky molecules. In contrast, shape-selective reactions suffer from reduced selectivity for the main product, but have the benefits of enhanced stability and activity. Selectivity can be promoted by increasing the intracrystalline mesoporosity and masking or eliminating the active sites on the external surface of the catalyst. Hierarchical zeolites perform better in reactions of bulky substrates, because of the bimodal textural properties of these zeolitic structures. However, imperfect connectivity between mesopores, a reduction in the strength of acid sites, and a larger amount of framework defects (even with a large mesopore surface area) decrease the catalytic activities of hierarchical zeolites. The results of previous studies show that higher catalytic activities and longer catalytic lifetimes are achieved for mesoporous and nanosized zeolites in liquid- and gas-phase reactions, respectively. For shape-selective reactions in the gaseous phase, the selectivity decreases, and the cracking efficiency of large reactants and selectivity in methanol transformation increase. The length of the micropore channel should therefore be compatible with the active sites present on the external surface of the catalyst, to enhance the catalytic performance.

\section{Industrial and economic aspects of preparation of nanosized and hierarchical zeolites}

Zeolite catalysts have a wide range of industrial applica- 
tions, and therefore the development of zeolite catalysts that give improved yields in industrial reactions is important. There are several methods for producing nanosized and hierarchical zeolites; the main question is which of these routes is appropriate in terms of economic and environmental demands. The exploration of appropriate synthetic procedures for nanostructured materials with various particle shapes and sizes is important in nanotechnology and nanoscience. The development of strategies that reduce the use of SDAs or solvents, and time and energy consumption, while affording high yields, is a priority. One important drawback during the synthesis of nanocrystals is the imperfect conversion of aluminosilicate species (especially silica precursors) to zeolite nanocrystallites, because of the use of dilute gels in batch synthetic systems; this makes scaling-up difficult. Additionally, recycling of wastes for use as new substrates for preparing new nanosized zeolites needs extra filtration and hydrothermal stages [139]. The operating efficiencies of continuous-flow microfluidic systems are better than those of conventional processes. Laboratory-scale reactions must go through three vital phases before scaling-up to the large-scale commercial stage. The first phase is evaluation of the mass transport characteristics, kinetics, and fluid hydrodynamics of the laboratory-scale reaction to clarify the process mechanism and enable condition optimization. The second and the third phases are related to optimization of factors that affect increased production in the pilot plant and plant, respectively. Clearly, any change in the reactor volume changes mass and heat transfers and affects the end-product quality. Microfluidic techniques, in contrast to traditional processes, which focus on the use of larger reactors to accommodate larger amounts of reactants, use a large number of the units that have already been optimized on the laboratory scale. Such techniques avoid the drawbacks of the large-volume reactors used in traditional scale-up methods. Microreactors have the benefits of rapid optimization of the experimental factors, simultaneous characterization of nanostructured products, control of the reaction parameters such as temperature gradient, interfacial contact, and residence time of the reactants, and simple automation [140]. For example, Pan et al. [69] produced nanosized silicalite crystals in 11 min using a pressurized capillary microreactor with an inner diameter of $1 \mathrm{~mm}$. This system overcame the restricted mass transfer caused by organic template cations around the colloidal particles. Silicalite nanocrystals (ca. $102.2 \mathrm{~nm}$ ) with $70 \%$ crystallinity were successfully synthesized at $150{ }^{\circ} \mathrm{C}$ and 0.7 MPa. The product crystallinity reached $100 \%$, with a mean particle size of $166.4 \mathrm{~nm}$, when the stainless-steel vessel was pressurized to $1 \mathrm{MPa}$ (synthesis solutions initially aged at 100 ${ }^{\circ} \mathrm{C}$ for $2 \mathrm{~h}$ ). The other controversial issue in handling nanosized zeolitic structures is their separation from the reaction medium. High-speed centrifugation and ultrasonic baths are usually used to redisperse the crystals. Flocculating agents such as polymers can be useful in preventing aggregation of nanocrystals to be filtered, but this introduces additional steps before obtaining the final product [139]. An alternative, less expensive diafiltration set-up was used to purify a nano-BEA zeolite [141]. During diafiltration, which is similar to both classic filtration and dialysis, samples are circulated through a membrane several times by pumping fresh solvent (water) to compensate for the reduction in the solution volume caused by passing through the filter. In this case, contaminants such as SDA (TEA+), soluble silicates, and $\mathrm{OH}^{-}$are present in the filtrate, and pure zeolite nanocrystals are suspended in water. This suspension is devoid of impurities and can be dehydrated and dried to give pure zeolite powder. This method removes the need for periodic isolation of the solid from the liquid phase and therefore saves energy and time. Theoretically, every zeolitic structure can be transformed into a hierarchical structure, therefore there is a wide range of potential novel applications of hierarchical zeolites, such as metal-phase carriers, ion-exchangers, and catalysts [142]. In recent years, much research has been performed on the synthesis and characterization of mesoporous zeolites, but their industrial applications are still restricted. The main reason is the large content of organic templates, which raises environmental issues and makes manufacturing expensive. Since the 1970s, the hierarchical zeolites used in industrial applications have mainly been prepared via acid or steam-assisted dealumination post-treatment methods. However, as the use of hierarchical zeolites becomes more widespread, other effective synthetic methods for mesoporous zeolites with potential industrial uses will be developed $[23,142,143]$. The requirements of large-scale synthesis and shaping of hierarchical zeolite catalysts need to be considered [97]. Recently, van Donk's group [23] summarized the hydrothermal flexibilities and stabilities, health/safety/environment issues, costs, and other topics related to mesoporous zeolite production (Table 3). Among the various methods for mesoporous zeolite synthesis, desilication is an efficient strategy because of its simplicity and cost effectiveness (Fig. 22).

Use of the desilication method in large-scale zeolite preparation was investigated by Groen and coworkers [143] by adjusting the mesoporosities in synthesized, and powdered and extruded commercial ZSM-5 zeolites ( $\mathrm{Si} / \mathrm{Al}=15-1000)$, on gram and kilogram scales. The scaled-up alkaline treatments of zeolite powders and extrudates were performed in two commercial set-ups, i.e., 0.6- and 6-L reactors, using various $\mathrm{NaOH}$ concentrations. The same high mesopore surface areas and reductions in micropore volume as in laboratory-scale experiments were observed after a short reaction time. The authors suggested that because the desilication treatment is chemically controlled, the process can be expanded from the laboratory scale. Zeolite extrudates also underwent alkaline treatment. Initially, probably because of the presence of a meso-macroporous binder in the shaped samples, a high mesopore surface area of $120 \mathrm{~m}^{2} / \mathrm{g}$ was observed. However, an increase in the mesopore surface area (120 to $180 \mathrm{~m}^{2} / \mathrm{g}$ ) and decrease in the micropore volume (from 0.12 to $0.08 \mathrm{~cm}^{3} / \mathrm{g}$ ) occurred under alkaline treatment using $\mathrm{NaOH}$ solution $(0.8$ mol/L). Because of the presence of a binder (mostly alumina, which is resistant to the alkaline process) and negligible dissolution of zeolite crystals, the extrudate samples were more resistant to desilication and less particle homogeneous rigidity was observed. The experimental results showed that $\mathrm{NaOH}$ solution was a better medium for mesoporosity creation than 
Table 3

Overview of advantages and disadvantages of methods for hierarchical zeolite synthesis. Reprinted with permission from Ref. [23]. Copyright 2011, John Wiley and Sons.

\begin{tabular}{|c|c|c|c|c|c|c|c|}
\hline & Formation Route & Production Cost & $\begin{array}{l}\text { HSE } \\
\text { issues }\end{array}$ & $\begin{array}{l}\text { Hypirothermal } \\
\text { stability }\end{array}$ & $\begin{array}{l}\text { Flexibility } \\
\text { in SVAI }\end{array}$ & $\begin{array}{l}\text { Predestined mesoporous } \\
\text { architecture }\end{array}$ & $\begin{array}{l}\text { Breakthrough develop- } \\
\text { ment expected }\end{array}$ \\
\hline Destructive & $\begin{array}{l}\text { dealumination } \\
\text { desilication }\end{array}$ & $\begin{array}{c}\text { low (if zeolite without } \\
\text { template) } \\
\text { low (if zeolite without } \\
\text { template) }\end{array}$ & no & $\begin{array}{l}\text { high } \\
\text { high }\end{array}$ & $\begin{array}{l}\text { medium } \\
\text { little }\end{array}$ & $\begin{array}{l}\text { no } \\
\text { no }\end{array}$ & $\begin{array}{l}\text { no } \\
\text { no }\end{array}$ \\
\hline Constructive & $\begin{array}{l}\text { zeolitization of mesopo- } \\
\text { rous material } \\
\text { soft templating } \\
\text { hard templating } \\
\text { mesostructuring of zeo- } \\
\text { lites }\end{array}$ & $\begin{array}{l}\text { high } \\
\text { medium/high } \\
\text { high } \\
\text { high }\end{array}$ & $\begin{array}{l}\text { yes } \\
\text { yes } \\
\text { yes } \\
\text { yes }\end{array}$ & $\begin{array}{c}\text { low } \\
\text { medium/high } \\
\text { high } \\
\text { medium/high }\end{array}$ & $\begin{array}{l}\text { medium } \\
\text { yes } \\
\text { yes } \\
\text { yes }\end{array}$ & $\begin{array}{c}\text { yes } \\
\text { yes/medium } \\
\text { medium } \\
\text { yes/medium }\end{array}$ & $\begin{array}{l}\text { medium } \\
\text { yes } \\
\text { no } \\
\text { yes }\end{array}$ \\
\hline
\end{tabular}

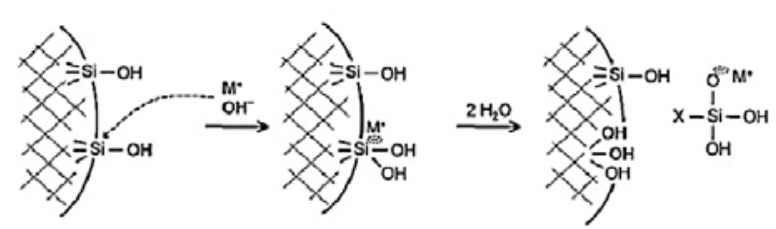

$\mathrm{M}^{+}=$hydrated $\mathrm{Li}^{+}, \mathrm{Na}^{+}$, or $\mathrm{K}^{+} ; \mathrm{X}=\mathrm{OH}$ or siloxane chain

Fig. 22. Si hydrolysis from zeolite framework in alkaline medium. Reprinted with permission from Ref. [143]. Copyright 2007, American Chemical Society.

$\mathrm{LiOH}$ and $\mathrm{KOH}$ solutions. The Si/Al ratio in the framework affected the alkaline treatment, but factors such as the time, temperature, stirring speed, and particle size had no significant effects. The most important outcome was preservation of the microporosity and acidity of the parent zeolite. Pérez-Ramírez et al. [144] recently prepared hierarchically structured MFI zeolites through desilication in laboratory-scale, medium-scale, and pilot set-ups for the first time (Fig. 23). The laboratory-scale treatment was performed at $338 \mathrm{~K}$ for $30 \mathrm{~min}$ using $\mathrm{NaOH}$ solution $(0.2 \mathrm{~mol} / \mathrm{L})$. The $\mathrm{N}_{2}$ adsorption-desorption iso-

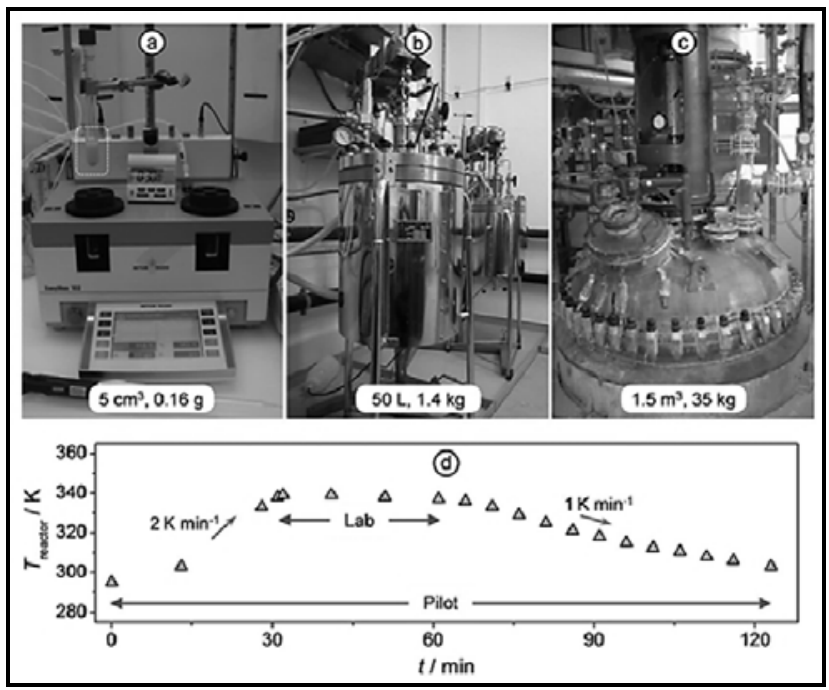

Fig. 23. (a) Laboratory-, (b) medium-, and (c) pilot-scale set-ups used for zeolite desilication, and (d) their temperature profiles. Reprinted with permission from Ref. [144]. Copyright 2011, John Wiley and Sons. therm of the product showed type-IV and type-I behaviors, corresponding to meso- and micro-porous systems, respectively, in the structure. Compared with the initial sample, the micropore volume decreased slightly, and the mesopore surface area was 3.5 times larger (Fig. 24). Hierarchical catalysts with the same porosity and composition were obtained on the pilot scale by treating $35 \mathrm{~kg}$ of the parent sample in a $1.5 \mathrm{~m}^{3}$ reactor. Similar results were obtained for desilication in a $50 \mathrm{~L}$ reactor (medium scale).

Catalytic conversion reactions involving polyethylene pyrolysis and alkylation of benzene with ethylene and benzyl alcohol were performed to assess the performances of the synthesized mesoporous pilot-scale zeolites. The performances of the mesoporous zeolites (pilot-scale), i.e., 100\% conversion after 15 min for benzyl alcohol and four-fold enhancement in ethylbenzene generation during alkylation of benzene with ethylene, and shortened light-off temperature in polyethylene pyrolysis, were better than those of conventional zeolites. Next, zeolite granules of size $2-5 \mathrm{~mm}$ were prepared using $20 \mathrm{wt} \%$ attapulgite (a binder with a needle-like morphology). The $\mathrm{N}_{2}$ adsorption results show that the zeolite pores were not occupied by the binder and the observed reduction in the mesopore surface area $\left(205 \mathrm{~m}^{2} / \mathrm{g}\right)$ was caused by the presence of attapulgite binder, which has a low porosity, and there were no

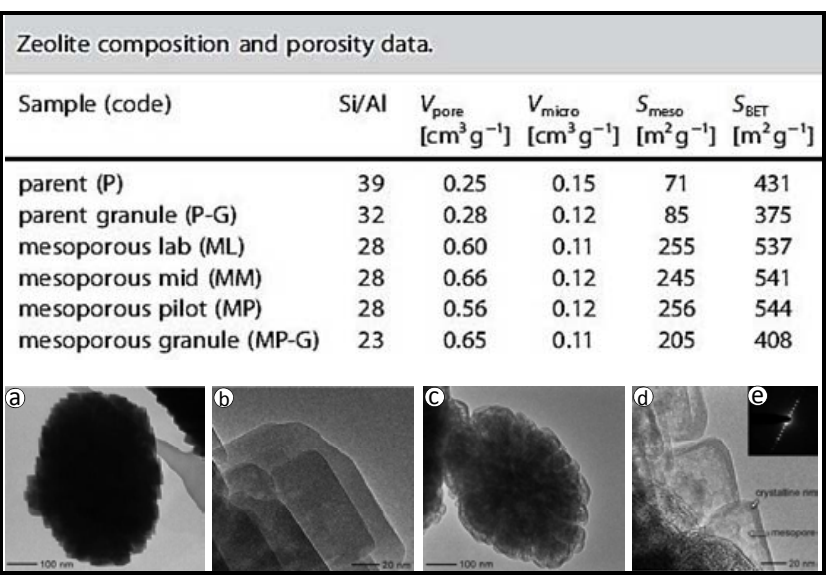

Fig. 24. Zeolite characterization and TEM images of $(a, b)$ parent zeolite (sample P) and (c, d) pilot-scale desilicated product (MP); (e) Selected-area electron diffraction pattern of crystal rim. Reprinted with permission from Ref. [144]. Copyright 2011, John Wiley and Sons. 
serious changes in the morphological properties.

Although the use of desilication has many benefits, zeolite content is lost because of dissolution of some of the material during alkaline treatment. It is essential to address this issue. Verboekend and Pérez-Ramírez [139] investigated the sustainability of manufacturing mesoporous zeolites via desilication and suggested actions such as increasing the reactor productivity, recycling the wastewater stream, organic, and dissolved species, and minimizing the separation steps (Fig. $25)$. The yields obtained in commercial zeolite syntheses using batch systems are generally about 100-250 g of final product per liter of starting materials. However, when alkaline treatment is performed using $33 \mathrm{~g}$ of parent zeolite in $1 \mathrm{~L}$ of basic solution, usually $0.2-\mathrm{mol} / \mathrm{L} \mathrm{NaOH}$, the amount of mesoporous zeolite obtained is $23 \mathrm{~g} / \mathrm{L}$. The reactor productivity can be improved by changing the $\mathrm{NaOH}$ concentration and using additives such as PDAs. An intrinsic feature of zeolite desilication is leaching of some of the crystalline zeolite into the liquid medium. It has been suggested that pure amorphous ordered mesoporous materials or composites of these materials and hierarchical zeolites can be obtained, using cationic surfactants as PDAs, from the recycled filtrate from alkaline treatment. It should be noted that the recrystallization of dissolved crystals cannot happen spontaneously because of the low concentration of $\mathrm{Si}$ species and moderate time and temperature conditions, unless pre-prepared zeolites are subjected to hydrothermal treatment in an SDA-containing basic medium. However, this second hydrothermal synthesis is different from the conventional one, and requires higher amounts of salts such as $\mathrm{NaCl}$ or $\mathrm{NaBr}$, because a higher salinity results in greater silica dissolution. If the relationships among $\mathrm{pH}$, salinity, and the zeolite content are adjusted appropriately, alkaline waste recovery is simple.

Another strategy for industrial desilication is to decrease the number of preparative stages. One step, which is usually

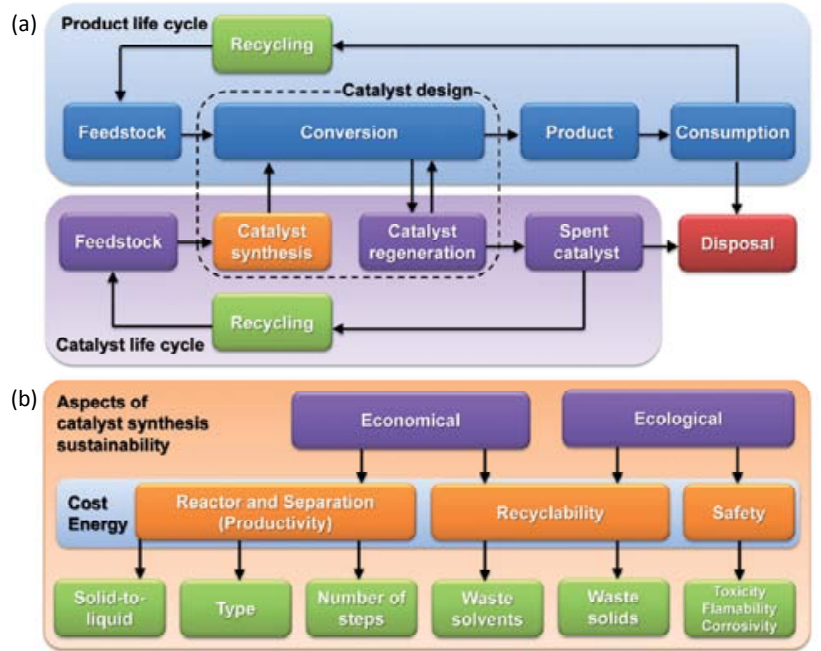

Fig. 25. (a) The basis of catalyst design (dashed box) usually depends on the product lifecycle (top), and the catalyst lifecycle (bottom) significantly contributes to sustainable chemistry. (b) Main aspects of sustainable manufacture of catalysts. Reprinted with permission from Ref. [139]. Copyright 2014, John Wiley and Sons. necessary in processes using $\mathrm{NaOH}$ solution, is to exchange $\mathrm{Na}$ cations, which have already replaced extra-framework cations in the zeolitic structure, to obtain the zeolite in the active protonic form. If an organic base solution that does not contain $\mathrm{Na}$ cations is used instead of $\mathrm{NaOH}$, this ion-exchange step can be omitted. The use of simple separation techniques such as sedimentation and then depletion of the supernatant can also reduce the number of experimental steps. As mentioned before, the low productivity of conventional batch reactors is one of the main drawbacks in process scale-up. Switching to continuous-mode reactors, especially with incorporation of continuous-mode separation techniques, can increase the efficiency compared with those of ordinary systems. Continuous-mode synthesis of microporous zeolites has been known for many years, but a two-fold productivity increase, from 1 to 100 $\mathrm{g} /(\mathrm{L} \cdot \mathrm{min})$, in continuous-mode manufacture of mesoporous zeolites by desilication has recently been reported. Continuous-mode synthesis of hierarchical zeolites also allows in-line shaping, particularly using extrusion or spray-drying (granulation) methods, and precludes the need for extra separation steps.

\section{Conclusions and prospects}

Because of their unusual and specific properties, molecular sieves, especially zeolites, have for many years attracted much attention in the fields of catalysis, ion-exchange, and sorption. Although conventional zeolites with microporous structures are very successful in processing small molecules, they are unsuitable for use with bulky substrates. To overcome this limitation, new porous materials with larger pores, such as mesoporous silica and metal-organic frameworks, have been synthesized. However, these mesoporous structures, which have amorphous walls, suffer from low hydrothermal stabilities; hydrothermal stability is an essential factor in many industrial processes involving high-temperature reactions. Another solution is to improve the zeolite performance by preparing their nanosized and hierarchical forms. Several strategies have been developed and new commercial mesoporous zeolites have become available. In this review, we have briefly discussed the synthetic methods and catalytic applications of such zeolites. According to the literature, zeolitic structures with larger pores and/or smaller crystallites give better performances, and it is anticipated that template-free synthetic routes such as desilication/recrystallization will be used industrially in the future. It is worth mentioning that in addition to the many benefits of nanozeolites with solely microporous system in the processing of bulky molecules on their external surfaces, the yields of reactions occurring inside their micropores, such as catalytic conversion of naphtha, and MTH, oxygen-containing chemicals to olefins, methanol to gasoline, and methanol to DME conversions, are increased because of the shorter diffusion pathways, arising from the crystal size, for the reactant/product hydrocarbons, and prevention of excessive reactions $[75,138]$. However, it is essential to note that larger pore systems should not be created in traditional zeolite frameworks at the expense of loss of their intrinsic features, 
e.g., hydrothermal stability. It is also critical to study the changes in acidity, hydrophobicity/hydrophilicity, and other properties of both types of zeolite. Moreover, in practical applications of nano and hierarchical zeolites, the choice of an appropriate binder for shaping powder zeolite products, without affecting the important functions of hierarchical zeolites, and the introduction of novel strategies instead of high-speed centrifugation methods for handling nanosized zeolites should be considered.

\section{References}

[1] S. Kulprathipanja, Zeolites in Industrial Separation and Catalysis, Wiley-VCH, Weinheim, 2010.

[2] M. Anbia, A. Habibi Davijani, Chem. Eng. J., 2013, 223, 899-907.

[3] Z. P. Wang, J. H. Yu, R. R. Xu, Chem. Soc. Rev., 2012, 41, 1729-1741.

[4] M. Anbia, F. Mohammadi Nejati, M. Jahangiri, A. Eskandari, V. Garshasbi, J. Sci. I. R. Iran, 2015, 26(3), 213-222.

[5] M. Anbia, Z. Parvin, Chem. Eng. Res. Des., 2011, 89, 641-647.

[6] F. Bandarchian, M. J. Anbia, Nat. Gas Sci. Eng., 2015, 26, 1380-1387.

[7] C. Martínez, J. Perez-Pariente, Zeolites and Ordered Porous Solids: Fundamentals and Applications, Editorial Universitat Politècnica de València, Madrid, 2011.

[8] R. M. Mohamed, H. M. Aly, M. F. El-Shahat, I. A. Ibrahim, Microporous Mesoporous Mater., 2005, 79, 7-12.

[9] M. Moliner, F. Rey, A. Corma, Angew. Chem. Int. Ed., 2013, 52, 13880-13889.

[10] B. Liu, Y. H. Zheng, N. Hu, T. Gui, Y. Q. Li, F. Zhang, R. F. Zhou, X. S. Chen, H. Kita, Microporous Mesoporous Mater, 2014, 196, 270-276.

[11] H. Y. Zhang, B. Xie, X. J. Meng, U. Müller, B. Yilmaz, M. Feyen, S. Maurer, H. Gies, T. Tatsumi, X. H. Bao, W. P. Zhang, D. De Vos, F. S. Xiao, Microporous Mesoporous Mater., 2013, 180, 123-129.

[12] W. J. Roth, J. Čejka, Catal. Sci. Technol., 2011, 1, 43-53.

[13] A. W. Chester, E. G. Derouane, Zeolite Characterization and Catalysis, Springer, New York, 2009.

[14] P. Misaelides, Microporous Mesoporous Mater., 2011, 144, 15-18.

[15] Y. I. Tarasevich, V. V. Goncharuk, V. E. Polyakov, D. A. Krysenko, Z. G. Ivanova, E. V. Aksenenko, M. Yu. Tryfonova, J. Ind. Eng. Chem., 2012, 18, 1438-1440.

[16] A. C. de Campos Bernardi, P. P. A. Oliviera, M. B. de Melo Monte, F. Souza-Barros, Microporous Mesoporous Mater., 2013, 167, 16-21.

[17] M. W. Ackley, S. U. Rege, H. Saxena, Microporous Mesoporous Mater., 2003, 61, 25-42.

[18] D. Papaioannou, P. D. Katsoulos, N. Panousis, H. Karatzias, Mi- croporous Mesoporous Mater., 2005, 84, 161-170.

[19] Y. Li, J. H. Yu, Chem. Rev., 2014, 114, 7268-7316.

[20] A. Seidel, M. Bickford, Kirk-Othmer Encyclopedia of Chemical Technology, John Wiley \& Sons, Inc., Hoboken, 2013.

[21] D. Serrano, J. Aguado, J. Escola, in: J. J. Spivey, Y. F. Han, K. M. Dooley eds, Catalysis, Vol. 23, RSC Publishing, Cambridge, 2011, 53.

[22] M. Anbia, M. Lashgari, Chem. Eng. J., 2009, 150, 555-560.

[23] R. Chal, C. Gérardin, M. Bulut, S. van Donk, ChemCatChem, 2011, 3, 67-81.

[24] L. Tosheva, V. P. Valtchev, Chem. Mater., 2005, 17, 2494-2513.

[25] M. Zaarour, B. Dong, I. Naydenova, R. Retoux, S. Mintova, Microporous Mesoporous Mater., 2014, 189, 11-21.

[26] V. Valtchev, L. Tosheva, Chem. Rev., 2013, 113, 6734-6760.

[27] K. K. Zhu, J. M. Sun, J. Liu, L. Q. Wang, H. Y. Wan, J. Z. Hu, Y. Wang, C. H. F. Peden, Z. M. Nie, ACS Catal., 2011, 1, 682-690.

[28] G. T. Vuong, V. T. Hoang, D. T. Nguyen, T. O. Do, Appl. Catal. A, 2010, 382, 231-239..

[29] X. Y. Chen, K. Wendell, J. Zhu, J. L. Li, X. X. Yu, Z. J. Zhang, Bioresour. Technol., 2012, 110, 79-85.

[30] G. T. Vuong, T. O. Do, J. Am. Chem. Soc., 2007, 129, 3810-3811.

[31] W. Song, R. E. Justice, C. A. Jones, V. H. Grassian, S. C. Larsen, Langmuir, 2004, 20, 4696-4702.

[32] S. Mintova, J. P. Gilson, V. Valtchev, Nanoscale, 2013, 5, 6693-6703.

[33] L. Burel, A. Tuel, Microporous Mesoporous Mater., 2013, 174, 90-99.

[34] N. Esmaeili, H. Kazemian, D. Bastani, Iran. J. Chem. Chem. Eng., 2011, 30, 9-14.

[35] B. Xie, J. W. Song, L. M. Ren, Y. Y. Ji, J. X. Li, F. S. Xiao, Chem. Mater., 2008, 20, 4533-4535.

[36] G. Reding, T. Mäurer, B. Kraushaar-Czarnetzki, Microporous Mesoporous Mater., 2003, 57, 83-92.

[37] D. Serrano, J. Aguado, J. Rodriguez, A. Peral, Stud. Surf. Sci. Catal., 2007, 170, 282-288.

[38] Y. S. Li, W. S. Yang, J. Membrane Sci., 2008, 316, 3-17.

[39] Z. W. Chen, S. Li, Y. Yan, Chem. Mater., 2005, 17, 2262-2266.

[40] M. Mehdipourghazi, A. Moheb, H. Kazemian, Microporous Mesoporous Mater., 2010, 136, 18-24.

[41] Y. Y. Hu, C. Liu, Y. H. Zhang, N. Ren, Y. Tang, Microporous Mesoporous Mater., 2009, 119, 306-314.

[42] Y. M. Liu, W. Huang, Y. S. Zhao, T. Dou, React. Kinet. Catal. Lett., 2009, 96, 157-163.

[43] M. Nasrollahzadeh, A. Ehsani, A. Rostami-Vartouni, Ultrason. Sonochem., 2014, 21, 275-282.

[44] H. Zhao, G. A. Baker, J. Chem. Technol. Biotechnol., 2013, 88, 3-12.

[45] X. J. Wang, C. L. Yan, Inorg. Mater., 2010, 46, 517-521.

\section{Graphical Abstract}

Chin. J. Catal., 2016, 37: 447-467 doi: 10.1016/S1872-2067(15)61038-5

\section{Nanosized and hierarchical zeolites: A short review}

Esmat Koohsaryan, Mansoor Anbia*

Iran University of Science and Technology, Iran

This review manuscript presents a short definition of zeolites, nanozeolites and hierarchical zeolites and discusses the recent research progress of the synthesis methods and catalytic applications of nanosized and mesoporous zeolites.

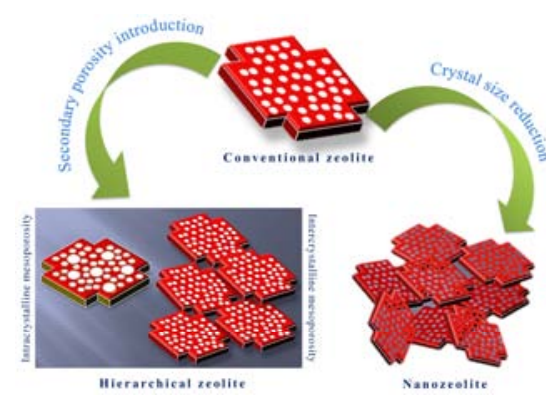


[46] R. Cai, Y. Liu, S. Gu, Y. Yan, J. Am. Chem. Soc., 2010, 132, 12776-12777.

[47] C. X. Zhao, L. He, S. Z. Qiao, A. P. Middelberg, Chem. Eng. Sci., 2011, $66,1463-1479$

[48] Y. C. Pan, M. H. Ju, J. F. Yao, L. X. Zhang, N. P. Xu, Chem. Commun., 2009, 7233-7235.

[49] T. Wakihara, A. Ihara, S. Inagaki, J. Tatami, K. Sato, K. Komeya, T. Meguro, Y. Kubota, A. Nakahira, Cryst. Growth. Des., 2011, 11, 5153-5158.

[50] T. Wakihara, R. Ichikawa, J. Tatami, A. Endo, K. Yoshida, Y. Sasaki, K. Komeya, T. Meguro, Cryst. Growth Des., 2011, 11, 955-958.

[51] A. Charkhi, H. Kazemian, M. Kazemeini, Powder Technol,, 2010, 203, 389-396.

[52] A. Nezamzadeh-Ejhieh, S. Khorsandi, J. Ind. Eng. Chem., 2014, 20, 937-946.

[53] G. T. Vuong, T. 0. Do, Microporous Mesoporous Mater., 2009, 120, 310-316

[54] J. Kecht, S. Mintova, T. Bein, Langmuir, 2008, 24, 4310-4315

[55] J. Kecht, S. Mintova, T. Bein, Microporous Mesoporous Mater., 2008, 116, 258-266.

[56] S. Mintova, V. Valtchev, T. Onfroy, C. Marichal, H. Knözinger, T. Bein, Microporous Mesoporous Mater., 2006, 90, 237-245.

[57] A. Sakthivel, A. Iida, K. Komura, Y. Sugi, K. V. R. Chary, Microporous Mesoporous Mater., 2009, 119, 322-330.

[58] M. Jafari, T. Mohammadi, M. Kazemimoghadam, Ceram. Int., 2014, 40, 12075-12080.

[59] M. Maldonado, M. D. Oleksiak, S. Chinta, J. D. Rimer, J. Am. Chem Soc., 2013, 135, 2641-2652.

[60] L. Zhang, S. L. Liu, S. J. Xie, L. Y. Xu, Microporous Mesoporous Mater. 2012, 147, 117-126.

[61] S. Gopalakrishnan, T. Yamaguchi, S. i. Nakao, J. Membrane Sci., 2006, 274, 102-107.

[62] E. P. Ng, J. M. Goupil, A. Vicente, C. Fernandez, R. Retoux, V. Valtchev, S. Mintova, Chem. Mater., 2012, 24, 4758-4765.

[63] N. Ren, J. Bronić, B. Subotić, Y. M. Song, X. C. Lü, Y. Tang, Mi croporous Mesoporous Mater, 2012, 147, 229-241.

[64] G. Majano, A. Darwiche, S. Mintova, V. Valtchev, Ind. Eng. Chem. Res., 2009, 48, 7084-7091.

[65] C. Madsen, C. Madsen, C. J. H. Jacobsen, Chem. Commun., 1999 673-674.

[66] I. Schmidt, C. Madsen, C. J. H. Jacobsen, Inorg. Chem., 2000, 39, 2279-2283.

[67] C. J. H. Jacobsen, C. Madsen, T. V. W. Janssens, H. J. Jakobsen, J. Skibsted, Microporous Mesoporous Mater., 2000, 39, 393-401.

[68] K. Tang, Y. G. Wang, L. J. Song, L. H. Duan, X. T. Zhang, Z. L. Sun, Mater. Lett., 2006, 60, 2158-2160.

[69] Y. C. Pan, J. F. Yao, L. X. Zhang, J. X. Ju, H. T. Wang, N. P. Xu, Chem. Eng. Technol., 2009, 32, 732-737.

[70] M. Rasouli, N. Yaghobi, M. Hafezi, M. Rasouli, J. Ind. Eng. Chem. 2012, 18, 1970-1976.

[71] S. K. H. Nejad-Darzi, A. Samadi-Maybodi, M. Ghobakhluo, J. Porous Mater., 2013, 20, 909-916.

[72] L. Seifi, A. Torabian, H. Kazemian, G. N. Bidhendi, A. A. Azimi, A. Charkhi, Water Air Soil Poll., 2011, 217, 611-625.

[73] F. Adam, J. T. Wong, E. P. Ng, Chem. Eng. J., 2013, 214, 63-67.

[74] X. M. Ji, W. Yao, Y. Y. Hu, N. Ren, J. Zhou, Y. P. Huang, Y. Tang, Sensor Mater, 2011, 23, 303-313.

[75] T. Tago, H. Konno, Y. Nakasaka, T. Masuda, Catal. Surv. Asia, 2012 $16,148-163$

[76] Y. M. Ni, A. M. Sun, X. L. Wu, G. L. Hai, J. L. Hu, T. Li, G. X. Li, Microporous Mesoporous Mater., 2011, 143, 435-442.

[77] S. G. Bao, G. Z. Liu, X. W. Zhang, L. Wang, Z. T. Mi, Ind. Eng. Chem.
Res., 2010, 49, 3972-3975.

[78] C. Covarrubias, F. Gracia, H. Palza, Appl. Catal. A, 2010, 384, 186-191.

[79] H. B. Zhang, Y. C. Ma, K. S. Song, Y. H. Zhang, Y. Tang, J. Catal., 2013, 302, 115-125.

[80] B. Louis, A. Vicente, C. Fernandez, V. Valtchev, J. Phys. Chem. C, 2011, 115, 18603-18610.

[81] H. Konno, T. Tago, Y. Nakasaka, R. Ohnaka, J. i Nishimura, T. Masuda, Microporous Mesoporous Mater., 2013, 175, 25-33.

[82] A. A. Rownaghi, F. Rezaei, J. Hedlund, Catal. Commun., 2011, 14, 37-41.

[83] Y. Huang, J. Ho, Z. Wang, P. Nakashima, A. J. Hill, H. Wang, Microporous Mesoporous Mater., 2009, 117, 490-496.

[84] Y. Zhang, N. Ren, Y. Tang, in: V. Valtchev, S. Mintova, M. Tsapatsis ed., Ordered Porous Solids: Recent Advances and Prospects, Elsevier, Amsterdam, 2008, 441.

[85] X. Y. Li, M. H. Sun, J. C. Rooke, L. H. Chen, B. L. Su, Chin. J. Catal, 2013, 34, 22-47.

[86] M. S. Holm, E. Taarning, K. Egeblad, C. H. Christensen, Catal. Today, 2011, 168, 3-16.

[87] D. P. Serrano, J. M. Escola, P. Pizarro, Chem. Soc. Rev., 2013, 42 , 4004-4035.

[88] J. Perez-Ramirez, C. H. Christensen, K. Egeblad, C. H. Christensen, J. C. Groen, Chem. Soc. Rev., 2008, 37, 2530-2542.

[89] Q. T. Sheng, K. C. Ling, Z. R. Li, L. F. Zhao, Fuel Process. Technol., 2013, 110, 73-78.

[90] R. Otomo, T. Yokoi, J. N. Kondo, T. Tatsumi, Appl. Catal. A, 2014, $470,318-326$

[91] K. Möller, T. Bein, Chem. Soc. Rev., 2013, 42, 3689-3707.

[92] M. D. González, Y. Cesteros, P. Salagre, Microporous Mesoporous Mater., 2011, 144, 162-170.

[93] R. Giudici, H. W. Kouwenhoven, R. Prins, Appl. Catal. A, 2000, 203, 101-110

[94] J. M. Müller, G. C. Mesquita, S. M. Franco, L. D. Borges, J. L. de Macedo, J. A. Dias, S. C. L. Dias, Microporous Mesoporous Mater. 2015, 204, 50-57.

[95] M. Müller, G. Harvey, R. Prins, Microporous Mesoporous Mater., 2000, 34, 135-147.

[96] J. Datka, J. Klinowski, B. Sulikowski, Catal. Lett., 1994, 25 , 403-404

[97] D. Verboekend, J. Pérez-Ramírez, Catal. Sci. Technol., 2011, 1, 879-890.

[98] A. Bonilla, D. Baudouin, J. Pérez-Ramírez, J. Catal., 2009, 265, 170-180.

[99] J. C. Groen, S. Abelló, L. A. Villaescusa, J. Pérez-Ramírez, Microporous Mesoporous Mater., 2008, 114, 93-102.

[100] J. C. Groen, J. C. Jansen, J. A. Moulijn, J. Pérez-Ramírez, J. Phys. Chem. B, 2004, 108, 13062-13065.

[101] S. Abello, A. Bonilla, J. Perez-Ramirez, Appl .Catal. A, 2009, 364, 191-198.

[102] K. H. Li, J. Valla, J. Garcia-Martinez, ChemCatChem, 2014, 6 , 46-66.

[103] D. P. Serrano, R. Sanz, P. Pizarro, I. Moreno, S. Shami, Microporous Mesoporous Mater., 2014, 189, 71-82.

[104] J. García-Martínez, M. Johnson, J. Valla, K. H. Li, J. Y. Ying, Catal. Sci. Technol., 2012, 2, 987-994.

[105] I. I. Ivanova, I. A. Kasyanov, A. A. Maerle, V. I. Zaikovskii, Microporous Mesoporous Mater., 2014, 189, 163-172.

[106] J. P. Na, G. Z. Liu, T. Y. Zhou, G. C. Ding, S. L. Hu, L. Wang, Catal. Lett., 2013, 143, 267-275.

[107] I. I. Ivanova, E. E. Knyazeva, Chem. Soc. Rev., 2013, 42 , 3671-3688. 
[108] N. D. Lysenko, V. G. Il'in, P. S. Yaremov, Theor. Exp. Chem., 2011, 47, 257-263.

[109] M. B. Yue, L. B. Sun, T. T. Zhuang, X. Dong, Y. Chun, J. H. Zhu, J. Mater. Chem., 2008, 18, 2044-2050.

[110] Y W. Zhang, T Okubo, M. Ogura, Chem. Commun., 2005, 2719-2720.

[111] Y. X. Jia, W. Han, G. X. Xiong, W. S. Yang, Mater. Lett., 2008, 62, 2400-2403.

[112] Y. J. Wang, Y. Tang, A. G. Dong, X. D. Wang, N. Ren, Z. Gao, J. Mater. Chem., 2002, 12, 1812-1818.

[113] Y. P. Guo, H. J. Wang, Y. J. Guo, L. H. Guo, L. F. Chu, C. X. Guo, Chem. Eng. J., 2011, 166, 391-400.

[114] G. Majano, S. Mintova, O. Ovsitser, B. Mihailova, T. Bein, Microporous Mesoporous Mater., 2005, 80, 227-235.

[115] D. P. Serrano, J. Aguado, G. Morales, J. M. Rodriguez, A. Peral, M. Thommes, J. P. Epping, B. F. Chmelka, Chem. Mater., 2009, 21, 641-654.

[116] K. Möller, B. Yilmaz, U. Müller, T. Bein, Chem. Mater., 2011, 23, 4301-4310.

[117] Y. M. Fang, H. Q. Hu, G. H. Chen, Chem. Mater., 2008, 20, 1670-1672.

[118] C. J. H. Jacobsen, C. Madsen, J. Houzvicka, I. Schmidt, A. Carlsson, J. Am. Chem. Soc., 2000, 122, 7116-7117.

[119] S. Frisch, L. M. Rösken, J. Caro, M. Wark, Microporous Mesoporous Mater., 2009, 120, 47-52.

[120] Y. C. Tong, T. B. Zhao, F. Y. Li, Y. Wang, Chem. Mater., 2006, 18, 4218-4220.

[121] K. K. Zhu, K. Egeblad, C. H. Christensen, Eur. J. Inorg. Chem., 2007, 3955-3960.

[122] I. Schmidt, A. Boisen, E. Gustavsson, K. Ståhl, S. Pehrson, S. Dahl, A. Carlsson, C. J. H. Jacobsen, Chem. Mater., 2001, 13, 4416-4418.

[123] H. B. Zhu, Z. C. Liu, Y. D. Wang, D. J. Kong, X. H. Yuan, Z. K. Xie, Chem. Mater., 2007, 20, 1134-1139.

[124] L. F. Wang, C. Y. Yin, Z. C. Shan, S. Liu, Y. C. Du, F. S. Xiao, Colloids Surf. A, 2009, 340, 126-130.

[125] F. J. Liu, T. Willhammar, L. Wang, L. F. Zhu, Q. Sun, X. J. Meng, W. Carrillo-Cabrera, X. D. Zou, F. S. Xiao, J. Am. Chem. Soc., 2012, 134, 4557-4560.

[126] F. S. Xiao, L. F. Wang, C. Y. Yin, K. F. Lin, Y. Di, J. X. Li, R. R. Xu, D. S.
Su, R. Schlögl, T. Yokoi, T. Tatsumi, Angew. Chem. Int. Ed., 2006, 45, 3090-3093.

[127] R. Srivastava, M. Choi, R. Ryoo, Chem. Commun., 2006, 4489-4491.

[128] L. F. Wang, Z. Zhang, C. Y. Yin, Z. C. Shan, F. S. Xiao, Microporous Mesoporous Mater., 2010, 131, 58-67.

[129] H. X. Tao, H. Yang, X. H. Liu, J. W. Ren, Y. Q. Wang, G. Z. Lu, Chem. Eng. J., 2013, 225, 686-694.

[130] H. X. Tao, C. L. Li, J. W. Ren, Y. Q. Wang, G. Z. Lu, J. Solid State Chem., 2011, 84, 1820-1827.

[131] J. Zhu, Y. H. Zhu, L. K. Zhu, M. Rigutto, A. van der Made, C. G. Yang, S. X. Pan, L. Wang, L. F. Zhu, Y. Y. Jin, Q. Sun, Q. M. Wu, X. J. Meng, D. L. Zhang, Y. Han, J. X. Li, Y. Y. Chu, A. M. Zheng, S. L. Qiu, X. M. Zheng, F. S. Xiao, J. Am. Chem. Soc., 2014, 136, 2503-2510.

[132] Q. Lü, G. Li, H. Y. Sun, Fuel, 2014, 130, 70-75.

[133] C. Yin, D. Tian, M. Xu, Y. J. Wei, X. Bao, Y. H. Chen, F. W. Wang, J. Colloid Interface Sci., 2013, 397, 108-113.

[134] C. Y. Yin, L. L. Feng, R. Ni, L. Y. Hu, X. Zhao, D. Tian, Powder Technol., 2014, 253, 10-13.

[135] J. S. Jin, C. Y. Peng, J. J. Wang, H. T. Liu, X. H. Gao, H. H. Liu, C. Y. Xu, Ind. Eng. Chem. Res., 2014, 53, 3406-3411.

[136] A. A. Rownaghi, F. Rezaei, M. Stante, J. Hedlund, Appl. Catal. B, 2012, 119, 56-61.

[137] A. A. Rownaghi, J. Hedlund, Ind. Eng. Chem. Res., 2011, 50, 11872-11878.

[138] Y. E. Yan, X. Guo, Y. H. Zhang, Y. Tang, Catal. Sci. Technol., 2015, 5, $772-785$.

[139] D. Verboekend, J. Pérez-Ramírez, ChemSusChem, 2014, 7, 753-764.

[140] P. R. Makgwane, S. S. Ray, J. Nanosci. Nanotechnol., 2014, 14, 1338-1363.

[141] R. R. Willis, A. I. Benin, Stud. Surf. Sci. Catal., 2007, 170, 242-249.

[142] D. Verboekend, S. Mitchell, J. Pérez-Ramírez, Chimia, 2013, 67, 327-332.

[143] J. C. Groen, J. A. Moulijn, J. Pérez-Ramírez, Ind. Eng. Chem. Res., 2007, 46, 4193-4201.

[144] J. Pérez-Ramírez, S. Mitchell, D. Verboekend, M. Milina, N. L. Michels, F. Krumeich, N. Marti, M. Erdmann, ChemCatChem, 2011, 3, 1731-1734.

\title{
纳米多级孔分子篮: 简短的综述
}

\author{
Esmat Koohsaryan, Mansoor Anbia* \\ 伊朗科技大学化学学院纳米孔材料研究室, 德黑兰16846-13114, 伊朗
}

摘要: 分子笁是一种三维微孔结构的硅铝酸盐晶体, 具有灵活多变的骨架和组成、较高的物理和水热稳定性、无毒、高比 表面积、离子可交换性以及很低的成本等特点, 因而在油品精制、石油化学、农业、水和污水处理等众多领域中用作离子 交换剂、催化剂和吸附剂. 尽管分子篮的应用是基于其本身的微孔结构, 但微孔也导致体积较大的反应物和产物分子的传 质阻力高. 通过制备纳米尺度和多级孔结构的分子篮等多种手段可克服常规分子篮所具有的传质限制. 人们已经开发了 多种方法制备了新型的分子篮材料, 并考察了它们在各种催化反应和吸附反应中的性能. 在反应体系中采用这种多级孔 的纳米分子篮, 有可能提高催化剂的使用寿命和催化性能, 抑制积碳和失活. 本综述概述了多级孔分子篎和纳米分子篮的 高性能及其合成方法的最新进展, 讨论了每个合成方法的优缺点, 简述了纳米分子篮和二级孔结构分子篮的催化应用, 并 与常规分子篮进行了比较.

关键词: 分子篮; 纳米分子篮; 多级分子篮; 催化剂

收稿日期: 2015-10-22. 接受日期: 2016-01-06. 出版日期: 2016-04-05.

*通讯联系人. 电话: +98-2177240068; 传真: +98-2177241026; 电子信箱: anbia@iust.ac.ir

本文的英文电子版由Elsevier出版社在ScienceDirect上出版(http://www.sciencedirect.com/science/journal/18722067). 